\title{
The Rankin-Selberg method for automorphic distributions
}

\author{
Stephen D. Miller ${ }^{\star}$ and Wilfried Schmid ${ }^{\star \star}$ \\ 1 Institute of Mathematics, The Hebrew University, Jerusalem 91904, Israel, and \\ Department of Mathematics, Hill Center-Busch Campus, Rutgers University, \\ Piscataway, NJ 08854 miller@math.huji.ac.il \\ 2 Department of Mathematics, Harvard University, Cambridge, MA 02138 \\ schmid@math.harvard.edu
}

\section{Introduction}

We recently established the holomorphic continuation and functional equation of the exterior square $L$-function for $G L(n, \mathbb{Z})$, and more generally, the archimedean theory of the $G L(n)$ exterior square $L$-function over $\mathbb{Q}$. We refer the reader to our paper 15 for a precise statement of the results and their relation to previous work on the subject. The purpose of this note is to give an account of our method in the simplest non-trivial cases, which can be explained without the technical overhead necessary for the general case.

Let us begin by recalling the classical results, about standard $L$-functions and Rankin-Selberg $L$-functions of modular forms. We consider a cuspidal modular form $F$, of weight $k$, on the upper half plane $\mathcal{H}$. To simplify the notation, we suppose that it is automorphic for $\Gamma=S L(2, \mathbb{Z})$, though the arguments can be adapted to congruence subgroups of $S L(2, \mathbb{Z})$. Like all modular forms, $F$ has a Fourier expansion,

$$
F(z)=\sum_{n \geq 1} a_{n} e(n z), \text { with } e(z)=_{\text {def }} e^{2 \pi i z} .
$$

For a general modular form, the Fourier series may involve a non-zero constant term $a_{0}$; it is the hypothesis of cuspidality that excludes the constant term. The Dirichlet series

$$
L(s, F)=\sum_{n \geq 1} a_{n} n^{-\frac{k-1}{2}-s}
$$

converges for Re $s \gg 0$, extends holomorphically to the entire $s$-plane, and satisfies a functional equation relating $L(s, F)$ to $L(1-s, F)$. This is the standard $L$-function of the modular form $F$.

\footnotetext{
* Partially supported by NSF grant DMS-0301172 and an Alfred P. Sloan Foundation Fellowship.

** Partially supported by NSF grant DMS-0500922
} 
Hecke proved the holomorphic continuation and functional equation by expressing $L(s, F)$ in terms of the Mellin transform of $F$ along the imaginary axis,

$$
\begin{aligned}
& \int_{0}^{\infty} F(i y) y^{s-1} d y=\sum_{n \geq 1} a_{n} \int_{0}^{\infty} e^{-2 \pi n y} y^{s-1} d y \\
& \quad=\sum_{n \geq 1} a_{n} n^{-s} \int_{0}^{\infty} e^{-2 \pi y} y^{s-1} d y=(2 \pi)^{-s} \Gamma(s) L\left(s-\frac{k-1}{2}, F\right),
\end{aligned}
$$

at least for Re $s \gg 0$. The transformation law for the modular form $F$ under $z \mapsto-1 / z$,

$$
F(-1 / z)=(-z)^{k} F(z),
$$

implies that $F(i y)$ decays rapidly not only as $y \rightarrow \infty$, but also as $y \rightarrow 0$. That makes the Mellin transform, and hence also $\Gamma\left(s+\frac{k-1}{2}\right) L(F, s)$, globally defined and holomorphic. The Gamma function has no zeroes, so $L(F, s)$ is entire as well. The transformation law 1.4, coupled with the change of variables $y \mapsto 1 / y$ and the shift $s \mapsto s+\frac{k-1}{2}$, gives the functional equation

$$
\begin{aligned}
& (2 \pi)^{-s-\frac{k-1}{2}} \Gamma\left(s+\frac{k-1}{2}\right) L(s, F)= \\
& \quad=i^{k}(2 \pi)^{s-1-\frac{k-1}{2}} \Gamma\left(1-s+\frac{k-1}{2}\right) L(1-s, F) .
\end{aligned}
$$

The factor $i^{k}$ comes up naturally in the computation, yet might be misleading since $\Gamma=S L(2, \mathbb{Z})$ admits only modular forms of even weights.

In addition to $F$, we now consider a second modular form of weight $k$, which need not be cuspidal,

$$
G(z)=\sum_{n \geq 0} b_{n} e(n z) .
$$

The Rankin-Selberg $L$-function of the pair $F, \bar{G}=$ complex conjugate of $G$, is the Dirichlet series

$$
L(s, F \otimes \bar{G})=\zeta(2 s) \sum_{n \geq 1} a_{n} \bar{b}_{n} n^{1-k-s} .
$$

Its analytic continuation and functional equation were established separately by Rankin [17 and Selberg [18]. The proof depends on properties of the nonholomorphic Eisenstein series

$$
\begin{aligned}
E_{s}(z)= & \pi^{-s} \Gamma(s) \zeta(2 s) \sum_{\gamma \in \Gamma_{\infty} \backslash \Gamma}(\operatorname{Im}(\gamma z))^{s} \\
& \left(\Gamma_{\infty}=\{\gamma \in \Gamma \mid \gamma \infty=\infty\}\right) .
\end{aligned}
$$

This sum is well defined since $\Gamma_{\infty}$ acts on $\mathcal{H}$ by integral translations. It converges for $\operatorname{Re} s>1$ and extends meromorphically to the entire $s$-plane with only one pole, of first order, at $s=1$. The function $E_{s}(z)$ is $\Gamma$-invariant by construction, has moderate growth as $\operatorname{Im} z \rightarrow \infty$, and satisfies the functional equation 


$$
E_{s}(z)=E_{1-s}(z) .
$$

Both $F(z)$ and $G(z)$ transform according to a factor of automorphy under the action of $\Gamma$, but $(\operatorname{Im} z)^{k} F(z) \bar{G}(z)$ is $\Gamma$-invariant, as is the measure $y^{-2} d x d y$. Since $G(z)$ and $E_{s}(z)$ have moderate growth as $\operatorname{Im} z \rightarrow \infty$, and since $F(z)$ decays rapidly, the integral

$$
I(s)=\int_{\Gamma \backslash \mathcal{H}}(\operatorname{Im} z)^{k-2} F(z) \bar{G}(z) E_{s}(z) d x d y
$$

converges. From $E_{s}(z)$, the function $I(s)$ inherits both the functional equation

$$
I(s)=I(1-s)
$$

and the analytic properties: it is holomorphic, with the exception of a potential first order pole at $s=1$.

The definition (1.8) of $E_{s}(z)$ involves a sum of $\operatorname{Im} \gamma z$, with $\gamma$ ranging over $\Gamma_{\infty} \backslash \Gamma$. But the rest of the integrand in 1.10 is $\Gamma$-invariant. That justifies the process known as "unfolding",

$$
\begin{aligned}
\pi^{s}( & \Gamma(s) \zeta(2 s))^{-1} I(s)= \\
& =\int_{\Gamma \backslash \mathcal{H}} \sum_{\gamma \in \Gamma_{\infty} \backslash \Gamma}(\operatorname{Im} z)^{k-2} F(z) \bar{G}(z)(\operatorname{Im}(\gamma z))^{s} d x d y \\
& =\int_{\Gamma_{\infty} \backslash \mathcal{H}}(\operatorname{Im} z)^{s+k-2} F(z) \bar{G}(z) d x d y,
\end{aligned}
$$

at least for Re $s>1$, in which case the integral on the right converges. Since $\Gamma_{\infty}$ acts on $\mathcal{H}$ by integral translations, the strip $\{0 \leq \operatorname{Re} z \leq 1\}$ constitutes a fundamental domain for this action. Substituting the series (1.1,1.6) for $F(z)$ and $G(z)$, one finds

$$
\begin{aligned}
\pi^{s}( & \Gamma(s) \zeta(2 s))^{-1} I(s)= \\
& =\int_{0}^{\infty} \int_{0}^{1} \sum_{\substack{n>0 \\
m \geq 0}} a_{n} \bar{b}_{m} e((n-m) x) e^{-2 \pi(n+m) y} y^{s+k-2} d x d y \\
& =\sum_{n \geq 1} a_{n} \bar{b}_{n} \int_{0}^{\infty} e^{-4 \pi n y} y^{s+k-2} d y \\
& =(4 \pi)^{-s-k+1} \Gamma(s+k-1) \sum_{n \geq 1} a_{n} \bar{b}_{n} n^{-s-k+1},
\end{aligned}
$$

again for $\operatorname{Re} s>1$. Equivalently,

$$
I(s)=2^{1-k}(2 \pi)^{1-k-2 s} \Gamma(s) \Gamma(s+k-1) L(s, F \otimes \bar{G}) .
$$

The Gamma factors have no zeroes, so $L(s, F \otimes \bar{G})$ extends holomorphically to all of $\mathbb{C}$, except possibly for a first order pole at $s=1$. In effect, 1.11 is the functional equation for the Rankin-Selberg $L$-function. With some additional 
effort one can modify these arguments, to make them work even when $F$ and $G$ have different weights.

Maass 12 extended the proofs of the analytic continuation and functional equation for the standard $L$-function to the case of Maass forms, i.e., $\Gamma$-invariant eigenfunctions of the hyperbolic Laplacian on $\mathcal{H}$; see section 2 below. Jacquet 6 treats the Rankin-Selberg $L$-function for Maass forms. We just saw how the Gamma factors in (1.3) and (1.13) arise directly from the standard integral representation of the Gamma function. In contrast, for Maass forms, the Gamma factor for the standard $L$-function arises from the Mellin transform of the Bessel function $K_{\nu}(y)$,

$$
\int_{0}^{\infty} K_{\nu}(y) y^{s-1}=2^{s-2} \Gamma\left(\frac{s-\nu}{2}\right) \Gamma\left(\frac{s+\nu}{2}\right) \quad(\operatorname{Re} s \gg 0),
$$

and for the Rankin-Selberg $L$-function of a pair of Maass forms, from the integral

$$
\begin{aligned}
\int_{0}^{\infty} & K_{\mu}(y) K_{\nu}(y) y^{s-1} d y= \\
& =2^{s-3} \frac{\Gamma\left(\frac{s-\mu-\nu}{2}\right) \Gamma\left(\frac{s+\mu-\nu}{2}\right) \Gamma\left(\frac{s-\mu+\nu}{2}\right) \Gamma\left(\frac{s+\mu+\nu}{2}\right)}{\Gamma(s)} \quad(\operatorname{Re} s \gg 0) .
\end{aligned}
$$

Though (1.16) can be established by elementary means, it is still complicated and its proof lacks a conceptual explanation.

In the case of Rankin-Selberg $L$-functions of higher rank groups, the integrals analogous to 1.16 become exceedingly difficult, or even impossible, to compute. In fact, it is commonly believed that such integrals may not always be expressible in terms of Gamma functions [1, §2.6]. If true, this would not contradict Langlands' prediction that the functional equations involve certain definite Gamma factors 10, 11]: the functional equations pin down only the ratios of the Gamma factors on the two sides, which can of course be expressed also as ratios of other functions.

Broadly speaking, the existing approaches to the $L$-functions for higher rank groups overcome the problem of computing these so-called archimedean integrals in one of two ways. Even if the integrals cannot be computed explicitly, it may be possible to establish a functional equation with unknown coefficients; it may then be possible to identify the coefficients in some special case, or by an analysis of their zeroes and poles. The Langlands-Shahidi method, on the other hand, often exhibits the functional equation with precisely the Gamma factors predicted by Langlands. Both methods have one difficulty in common: ruling out poles - other than those at the expected places - of the $L$-functions in question requires considerable effort, and is not always possible.

We are approaching the analytic continuation and functional equation of $L$-functions from a different point of view. Instead of working with automorphic forms - i.e., the higher dimensional analogues of modular forms and 
Maass forms - we attach the $L$-functions to automorphic distributions. In the case of modular forms and Maass forms, the automorphic distributions can be described quite concretely as boundary values. Alternatively but equivalently, they can be described abstractly; see $14, \S 2]$ or section three below. Computing with distributions presents some technical difficulties. What we gain in return are explicit formulas for the archimedean integrals that arise in the setting of automorphic distributions. This has led us to some new results.

In the next section we show how our method works in the simplest case, for the standard $L$-functions of modular forms and Maass forms. We treat the Rankin-Selberg $L$-function in section four, following the description of our main analytic tool in section three. Section five, finally, is devoted to the exterior square $L$-function for $G L(4, \mathbb{Z})$. That is the first not-entirely-trivial case of the main result of 15 . It can be explained more transparently than the general case for two reasons: the main analytic tool is the pairing of distributions, which for $G L(4)$ reduces to a variant of the Rankin-Selberg method for $G L(2)$. Also, the general case involves a somewhat subtle induction, with $G L(4)$ representing merely the initial step.

\section{Standard $L$-functions for $S L(2)$}

Holomorphic functions on the disk or the upper half plane have hyperfunction boundary values, essentially by definition of the notion of hyperfunction. Holomorphic functions of moderate growth, in particular modular forms, have distribution boundary values:

$$
\tau(x)=\lim _{y \rightarrow 0^{+}} F(x+i y)
$$

is the automorphic distribution corresponding to a modular form $F$ for $S L(2, \mathbb{Z})$, of weight $k$. The limit exists in the strong distribution topology. From $F$, the distribution $\tau$ inherits its $S L(2, \mathbb{Z})$-automorphy property

$$
\tau(x)=(c x+d)^{-k} \tau\left(\frac{a x+b}{c x+d}\right) \quad \text { for all } \quad\left(\begin{array}{ll}
a & b \\
c & d
\end{array}\right) \in S L(2, \mathbb{Z}) .
$$

In terms of Fourier expansion (1.1) of the cuspidal modular form $F(z)$, the limit 2.1) can be taken term-by-term,

$$
\tau(x)=\sum_{n>0} a_{n} e(n x) .
$$

We shall argue next that it makes sense to take the Mellin transform of the distribution $\tau$, and that this Mellin is an entire function of the variable $s$. The argument will be a special case of the techniques developed in our paper [13].

Note that the periodic distribution $\tau$ has no constant term. It can therefore be expressed as the $k$-th derivative of a continuous, periodic function $\phi_{k}$, for every sufficiently large integer $k$, 


$$
\begin{aligned}
\tau(x)= & \phi_{k}^{(k)}(x), \text { with } \phi_{k} \in C(\mathbb{R} / \mathbb{Z}) \\
& \left(\phi_{k}(x)=\sum_{n>0}(2 \pi i n)^{-k} a_{n} e(n x)\right) .
\end{aligned}
$$

Using the formal rule for pairing the "test function" $x^{s-1}$ against the derivative of a distribution, we find

$$
x^{s-1} \tau(x)=x^{s-1} \frac{d^{k}}{d x^{k}} \phi_{k}(x)=(-1)^{k} \phi_{k}(x) \frac{d^{k}}{d x^{k}} x^{s-1} .
$$

As a continuous, periodic function, $\phi_{k}$ is bounded. That makes the expression on the right in 2.5 integrable away from $x=0$, provided $k>\operatorname{Re} s$. Indeed, if we multiply the Mellin kernel $x^{s-1}$ by a cutoff function $\psi \in C^{\infty}(\mathbb{R})$, with $\psi(x) \equiv 1$ near $x=\infty$ and $\psi(x) \equiv 0$ near $x=0$, the resulting integral is an entire function of the variable $s$ - we simply choose $k$ larger than the real part of any particular $s$. Increasing the value of $k$ further does not affect the integral, as can be seen by a legitimate application of integration by parts. The identity 2.2 , with $a=d=0, b=-c=1$, gives

$$
\tau(x)=(-x)^{-k} \tau(-1 / x),
$$

so the behavior of $\tau(x)$ near zero duplicates its behavior near $\infty$, except for the factor $(-x)^{k}$ which can be absorbed into the Mellin kernel. The expression on the right in 2.5 is therefore integrable even down to zero, and

$$
s \mapsto \int_{0}^{\infty} \tau(x) x^{s-1} d x \text { is a well defined, entire holomorphic function. }
$$

The change of variables $x \mapsto 1 / x$ and the transformation law 2.6 imply

$$
\int_{0}^{\infty} \tau(x) x^{s-1} d x=(-1)^{k} \int_{0}^{\infty} \tau(-x) x^{k-s-1} d x .
$$

The integral on the right is of course well defined, for the same reason as the integral 2.7 .

In view of the argument we just sketched, it is entirely legitimate to replace $\tau(x)$ by its Fourier series and to interchange the order of integration and summation: for $\operatorname{Re} s \gg 0$,

$$
\begin{aligned}
& \int_{0}^{\infty} \tau(x) x^{s-1} d x=\int_{0}^{\infty} \sum_{n>0} a_{n} e(n x) x^{s-1} d x \\
& \quad=\sum_{n>0} a_{n} \int_{0}^{\infty} e(n x) x^{s-1} d x=L\left(s-\frac{k-1}{2}, F\right) \int_{0}^{\infty} e(x) x^{s-1} d x
\end{aligned}
$$

recall 1.2. The integral on the right makes sense for $\operatorname{Re} s>0$ if one regards $e(x)$ as a distribution and applies integration by parts, as was done in the case of $\tau(x)$. In the range $0<\operatorname{Re} s<1$ it converges conditionally. This integral is well known, 


$$
\int_{0}^{\infty} e(x) x^{s-1} d x=(2 \pi)^{-s} \Gamma(s) e(s / 4) \quad(0<\operatorname{Re} s<1) .
$$

Since $\Gamma(s) e(s / 4)$ has no zeroes, 2.7) and 2.92.10 imply that $L(s, F)$ is entire. Replacing $\tau(x)$ by $\tau(-x)$ in $(2.9)$ has the effect of replacing $e(x)$ by $e(-x)$, and accordingly the factor $e(s / 4)$ by $e(-s / 4)$ in 2.10). Thus 2.7 2.10 imply

$$
\begin{aligned}
& (2 \pi)^{-s} e(s / 4) \Gamma(s) L\left(s-\frac{k-1}{2}, F\right)= \\
& \quad=(-1)^{k}(2 \pi)^{s-k} e((s-k) / 4) \Gamma(k-s) L\left(1-s+\frac{k+1}{2}, F\right) .
\end{aligned}
$$

Since $e(-k / 4)=i^{-k}$, this functional equation is equivalent to the functional equation stated in (1.5).

A Maass form is a $\Gamma$-invariant eigenfunction $F \in C^{\infty}(\mathcal{H})$ for the hyperbolic Laplacian $\Delta$, of moderate growth towards the boundary of $\mathcal{H}$. It is convenient to express the eigenvalue as $\left(\lambda^{2}-1\right) / 4$, so that

$$
y^{2}\left(\frac{\partial^{2}}{\partial x^{2}}+\frac{\partial^{2}}{\partial y^{2}}\right) F=\frac{\lambda^{2}-1}{4} F .
$$

Near the real axis, the Maass form $F$ has an asymptotic expansion,

$$
F(x+i y) \sim y^{\frac{1-\lambda}{2}} \sum_{k \geq 0} \tau_{\lambda, k}(x) y^{2 k}+y^{\frac{1+\lambda}{2}} \sum_{k \geq 0} \tau_{-\lambda, k}(x) y^{2 k}
$$

as $y$ tends to zero from above, with distribution coefficients $\tau_{ \pm \lambda, k}$. In the exceptional case $\lambda=0$, the leading terms $y^{(1-\lambda) / 2}, y^{(1+\lambda) / 2}$ must be replaced by, respectively, $y^{1 / 2}$ and $y^{1 / 2} \log y$. The leading coefficients

$$
\tau_{\lambda}={ }_{\text {def }} \tau_{\lambda, 0}, \quad \tau_{-\lambda}==_{\text {def }} \tau_{-\lambda, 0}
$$

determine the others recursively. They are the automorphic distributions corresponding to the Maass form $F$. Each of the two also determines the otherin a way we shall explain later - unless $\lambda$ is a negative odd integer, in which case the $\tau_{-\lambda, k}$ all vanish identically. To avoid making statements with trivial counterexamples, we shall not consider $\tau_{-\lambda}$ when $\lambda \in \mathbb{Z}_{<0} \cap(2 \mathbb{Z}+1)$, and for $\lambda=0$, we shall only consider the coefficient of $y^{1 / 2}$, not the coefficient of $y^{1 / 2} \log y$.

Unlike modular forms, Maass forms are $\Gamma$-invariant as functions, i.e., without a factor of automorphy. However, because of the nature of the asymptotic expansion (2.13), the $\Gamma$-invariance of $F$ translates into an automorphy condition on the automorphic distributions,

$$
\tau_{\lambda}(x)=|c x+d|^{\lambda-1} \tau_{\lambda}\left(\frac{a x+b}{c x+d}\right) \quad \text { for all } \quad\left(\begin{array}{ll}
a & b \\
c & d
\end{array}\right) \in \Gamma .
$$

To simplify the discussion, we suppose $\Gamma=S L(2, \mathbb{Z})$, as before. Then 2.15), with $a=b=d=1, c=0$ implies $\tau_{\lambda}(x) \equiv \tau_{\lambda}(x+1)$, so $\tau_{\lambda}$ has a Fourier expansion 


$$
\tau_{\lambda}(x)=\sum_{n \in \mathbb{Z}} a_{n} e(n x) .
$$

From the point of view of $L$-functions, cuspidal Maass forms are more interesting than non-cuspidal forms. The condition of cuspidality on $F$ is equivalent to two conditions on the automorphic distribution $\tau_{\lambda}$, namely

$$
a_{0}=0, \text { and } \tau_{\lambda} \text { vanishes to infinite order at } x=0
$$

13. To explain the meaning of the second condition, we note that the discussion leading up to (2.4) applies also in the present context, since $a_{0}=0$. The automorphy condition (2.15), with $a=d=0, b=-c=-1$, asserts

$$
\tau_{\lambda}(x)=|x|^{\lambda-1} \tau_{\lambda}(-1 / x) .
$$

Combined with (2.4) and the chain rule for the change of variables $x \mapsto-1 / x$, this implies

$$
\tau_{\lambda}(x)=|x|^{\lambda-1}\left(x^{2} \frac{d}{d x}\right)^{k}\left(\phi_{k}(-1 / x)\right) \text { on } \mathbb{R}-\{0\},
$$

for every sufficiently large $k \in \mathbb{N}$, with some $\phi_{k} \in C(\mathbb{R}-\{0\})$ which remains bounded as $|x| \rightarrow \infty$. Moving the factor $|x|^{\lambda-1}$ across the differential operator and keeping track of the powers of $x$ shows that the right hand side of $(2.19)$ defines a distribution even on a neighborhood of the origin - a distribution with the remarkable property that for each $\ell \in \mathbb{N}$ it can be expressed, locally near $x=0$, as

$$
x^{\ell} P_{\ell}\left(x \frac{d}{d x}\right) \psi_{\ell}(x) \text {, with } \psi_{\ell} \text { defined and continuous near the origin; }
$$

here $P_{\ell}$ denotes a complex polynomial, whose coefficients depend on $\ell$ and $\lambda$. In [13] we introduced the terminology vanishing to infinite order at $x=0$ for the property (2.20) of a distribution defined on a neighborhood of the origin in $\mathbb{R}$.

To summarize the discussion so far, we have shown that a distribution $\tau_{\lambda}$ satisfying the automorphy condition $(2.15)$ for $\Gamma=S L(2, \mathbb{R})$, and additionally the condition $a_{0}=0$, agrees on $\mathbb{R}-\{0\}$ with a distribution that vanishes to infinite order at $x=0$. Thus either $\tau_{\lambda}$ itself vanishes to infinite order at $x=0-$ this is the meaning of the second condition in (2.17), of course - or else differs from such a distribution by one with support at the origin. A distribution supported at the origin is a linear combination of the delta function and its derivatives, and cannot vanish to infinite order at $x=0$ unless it is identically zero. If, contrary to our standing hypothesis, $\Gamma$ is a congruence subgroup of $S L(2, \mathbb{Z})$, the conditions 2.17 must be imposed at each of the cusps of $\Gamma$. In that case the second condition (2.17) must also be stated slightly differently.

If $F(x+i y)$ is a Maass form, then so is $F(-x+i y)$. It therefore makes sense to speak of even and odd Maass forms, i.e., Maass forms such that $F(-x+i y)= \pm F(x+i y)$. Every Maass form can be expressed uniquely as the sum of an even and an odd Maass form. If $F$ is cuspidal, then so are 
the even and odd parts. The parity of $F$ affects the Gamma factors in the functional equation of $L(s, F)$. We shall therefore suppose that $F$, and hence also $\tau_{\lambda}$, has a definite parity,

$$
\begin{aligned}
& \tau_{\lambda}(-x)=(-1)^{\eta} \tau_{\lambda}(x), \quad \text { or equivalently } \\
& \qquad a_{-n}=(-1)^{\eta} a_{n} \text { for all } n \quad(\eta \in \mathbb{Z} / 2 \mathbb{Z}) .
\end{aligned}
$$

We also suppose that $F$ is cuspidal, so that $\tau_{\lambda}$ satisfies (2.17). As one consequence of the parity condition,the $L$-function

$$
L(s, F)=\sum_{n \geq 1} a_{n} n^{-s+\frac{\lambda}{2}} \quad(\operatorname{Re} s \gg 0)
$$

completely determines all the $a_{n}$, and therefore also $\tau_{\lambda}$ and $F$. We had remarked earlier that $\tau_{\lambda}$ and $\tau_{-\lambda}$ play essentially symmetric roles unless $k$ is a negative odd integer or $\lambda=0$. Outside of those exceptional cases, the Fourier coefficients of $\tau_{\lambda}$ and $\tau_{-\lambda}$ are related by the factor of proportionality $c_{\lambda}|n|^{\lambda}$, with $c_{\lambda} \neq 0$. Switching $\tau_{\lambda}$ and $\tau_{-\lambda}$ has the minor effect of renormalizing the $L$-function 2.22 by the non-zero constant $c_{\lambda}$. It is not difficult to eliminate the remaining ambiguity in normalizing $L(s, F)$, but we shall not pursue the matter here.

Arguing exactly as in the case of a modular form, we see that the signed Mellin transform

$$
M_{\eta}\left(s, \tau_{\lambda}\right)=\int_{\mathbb{R}} \tau_{\lambda}(x)(\operatorname{sgn} x)^{\eta}|x|^{s-1} d x
$$

is a well defined entire holomorphic function. It is legitimate to substitute the Fourier series 2.16 for $\tau_{\lambda}$ and to interchange the order of summation and integration, again for the same reasons as in the case of modular forms, hence

$$
\begin{aligned}
M_{\eta}\left(s, \tau_{\lambda}\right) & =2 \sum_{n \geq 1} a_{n} n^{s} \int_{\mathbb{R}} e(x)(\operatorname{sgn} x)^{\eta}|x|^{s-1} d x \\
& =2 G_{\eta}(s) L\left(s+\frac{\lambda}{2}, F\right),
\end{aligned}
$$

with

$$
G_{\eta}(s)=\int_{\mathbb{R}} e(x)(\operatorname{sgn} x)^{\eta}|x|^{s-1} d x= \begin{cases}\frac{2 \Gamma(s)}{(2 \pi)^{s}} \cos \left(\frac{\pi s}{2}\right) & \text { if } \eta=0 \\ \frac{2 i \Gamma(s)}{(2 \pi)^{s}} \sin \left(\frac{\pi s}{2}\right) & \text { if } \eta=1\end{cases}
$$

the explicit formula for $G_{\eta}(s)$ follows from 2.10. Since $M_{\eta}(s, F)$ is entire, 2.242 .25 show that $L(s, F)$ extends meromorphically to the entire $s$-plane.

The change of variables $x \mapsto-1 / x$ in 2.23 , combined with the transformation rule 2.18, gives the functional equation

$$
M_{\eta}\left(s, \tau_{\lambda}\right)=(-1)^{\eta} M_{\eta}\left(1-s-\lambda, \tau_{\lambda}\right),
$$

which in turn implies the functional equation 


$$
G_{\eta}\left(s-\frac{\lambda}{2}\right) L(s, F)=(-1)^{\eta} G_{\eta}\left(1-s-\frac{\lambda}{2}\right) L(1-s, F)
$$

for $L(s, F)$. Standard Gamma identities establish the equivalence between Maass' version of the functional equation and (2.27).

Though we know that the product $G_{\eta}\left(s-\frac{\lambda}{2}\right) L(s, F)$ is entire, we cannot yet conclude that $L(s, F)$ is also entire: unlike $\Gamma(s), G_{\eta}(s)$ has zeroes. To deal with this problem, we consider the Fourier transform $\widehat{\tau}_{\lambda}$ of the tempered distribution $\tau_{\lambda}$. We use the normalization $\widehat{f}(y)=\int_{\mathbb{R}} f(x) e(-x y) d x$. Then $e(n x)$, considered as tempered distribution, has Fourier transform $\mathcal{F} e(n x)=$ $\delta_{n}(x)=$ Dirac delta function at $x=n$, and

$$
\widehat{\tau}_{\lambda}(x)=\sum_{n \neq 0} a_{n} \delta_{n}(x) .
$$

This distribution visibly vanishes in a neighborhood of the origin, in particular vanishes to infinite order at $x=0$. According to [13, theorem 3.19], the fact that $\tau_{\lambda}$ vanishes to infinite order at $x=0$ - cf. (2.17) - implies that $\widehat{\tau}_{\lambda}(1 / x)$ extends across the origin to a distribution that vanishes there to infinite order. Since both $\widehat{\tau}_{\lambda}(x)$ and $\widehat{\tau}_{\lambda}(1 / x)$ have this property, the signed Mellin transform

$$
\begin{aligned}
M_{\eta}\left(s, \widehat{\tau}_{\lambda}\right) & =2 \sum_{n>0} a_{n} n^{s-1} \quad(\operatorname{Re} s \ll 0) \\
& =2 L\left(1-s+\frac{\lambda}{2}, F\right)
\end{aligned}
$$

is a well defined, entire holomorphic function. In other words, $L(s, F)$ is entire, as was to be shown.

The preceding argument essentially applies also to the case of modular forms, except that one is then dealing with automorphic distributions that are neither even nor odd, but have only positive Fourier coefficients. In fact, if one considers modular forms and Maass forms not for $S L(2)$ but for $G L(2)$, a single argument treats both types of automorphic distributions absolutely uniformly. However, the case of modular forms is simpler in one important respect: the fact that the $L$-function has no poles requires no special argument.

\section{Pairings of automorphic distributions}

In the last section we encountered automorphic distributions as distributions on the real line, obtained by a limiting process. For higher rank groups, it is necessary to take a more abstract point of view, which we shall now explain.

Initially in this section $G$ shall denote a reductive Lie group, $Z_{G}^{0}$ the identity component in the center $Z_{G}$ of $G$, and $\Gamma \subset G$ an arithmetically defined subgroup. Note that $G$ acts unitarily on $L^{2}\left(\Gamma \backslash G / Z_{G}^{0}\right)$, via right translation. We consider an irreducible unitary representation $(\pi, V)$ of $G$ which occurs discretely in $L^{2}\left(\Gamma \backslash G / Z_{G}^{0}\right)$,

$$
j: V \hookrightarrow L^{2}\left(\Gamma \backslash G / Z_{G}^{0}\right) .
$$


Recall the notion of a $C^{\infty}$ vector for $\pi$ : a vector $v \in V$ such that $g \mapsto \pi(g) v$ is a $C^{\infty}$ map from $G$ to the Hilbert space $V$. The space of $C^{\infty}$ vectors $V^{\infty} \subset V$ is dense, $G$-invariant, and gets mapped to $C^{\infty}\left(\Gamma \backslash G / Z_{G}^{0}\right)$ by the embedding (3.1). That makes

$$
\tau=\tau_{j}: V^{\infty} \longrightarrow \mathbb{C}, \quad \tau(v)=j v(e),
$$

a well defined linear map. It is $\Gamma$-invariant because $j v \in C^{\infty}\left(\Gamma \backslash G / Z_{G}^{0}\right)$, and is continuous with respect to the natural topology on $V^{\infty}$. One should therefore think of $\tau$ as a $\Gamma$-invariant distribution vector for the dual representation $\left(\pi^{\prime}, V^{\prime}\right)$ - i.e., $\tau \in\left(\left(V^{\prime}\right)^{-\infty}\right)^{\Gamma}$. Very importantly, $\tau$ determines $j$ completely. Indeed, $j$ is $G$-invariant, so the defining identity 3.2 specifies the value of $j v, v \in V^{\infty}$, not only at the identity, but at any $g \in G$. Since $V^{\infty}$ is dense in $V$, knowing the effect of $j$ on $V^{\infty}$ means knowing $j$.

The space $L^{2}\left(\Gamma \backslash G / Z_{G}^{0}\right)$ is self-dual, hence if $V$ occurs discretely, so does its dual $V^{\prime}$. Since we shall be working primarily with $\tau$, we switch the roles of $V$ and $V^{\prime}$. From now on,

$$
\tau \in\left(V^{-\infty}\right)^{\Gamma}
$$

shall denote a $\Gamma$-invariant distribution vector corresponding to a discrete embedding $V^{\prime} \hookrightarrow L^{2}\left(\Gamma \backslash G / Z_{G}^{0}\right)$. Not all $\Gamma$-invariant distribution vectors correspond to embeddings into $L^{2}\left(\Gamma \backslash G / Z_{G}^{0}\right)$; some correspond to Eisenstein series, and others not even to those.

The arithmetically defined subgroup $\Gamma$ is arithmetic with respect to a particular $\mathbb{Q}$-structure on $G$. If $P \subset G$ is a parabolic subgroup, defined over $\mathbb{Q}$, with unipotent radical $U$, then $\Gamma \cap U$ is a lattice in $U$; in other words, the quotient $U /(\Gamma \cap U)$ is compact. One calls $\tau \in\left(V^{-\infty}\right)^{\Gamma}$ cuspidal if

$$
\int_{U /(\Gamma \cap U)} \pi(u) \tau d u=0,
$$

for the unipotent radical $U$ of any parabolic subgroup $P$ that is defined over $\mathbb{Q}$. Since there exist only finitely many $\Gamma$-conjugacy classes of such parabolics, cuspidality amounts to only finitely many conditions. Essentially by definition, cuspidal embeddings $V^{\prime} \hookrightarrow L^{2}\left(\Gamma \backslash G / Z_{G}^{0}\right)$ correspond to cuspidal distribution vectors $\tau \in\left(V^{-\infty}\right)^{\Gamma}$, and conversely every cuspidal automorphic $\tau$ arises from a cuspidal embedding of $V^{\prime}$ into $L^{2}\left(\Gamma \backslash G / Z_{G}^{0}\right)$.

To get a handle on $\tau \in\left(V^{-\infty}\right)^{\Gamma}$, we realize the space of $C^{\infty}$ vectors $V^{\infty}$ as a subspace $V^{\infty} \hookrightarrow V_{\lambda, \delta}^{\infty}$ of the space of $C^{\infty}$ vectors for a not-necessarilyunitary principal series representation $\left(\pi_{\lambda, \delta}, V_{\lambda, \delta}\right)$. The Casselman embedding theorem [3] guarantees the existence of such an embedding. For the moment, we leave the meaning of the subscripts $\lambda, \delta$ undefined. They are the parameters of the principal series, which we shall explain presently in the relevant cases. A theorem of Casselman-Wallach [3, 22] asserts that the inclusion $V^{\infty} \hookrightarrow V_{\lambda, \delta}^{\infty}$ extends continuously to an embedding of the space of distribution vectors,

$$
V^{-\infty} \hookrightarrow V_{\lambda, \delta}^{-\infty}
$$


This allows us to consider the automorphic distribution $\tau$ as a distribution vector for a principal series representation,

$$
\tau \in\left(V_{\lambda, \delta}^{-\infty}\right)^{\Gamma}
$$

When $G=S L(2, \mathbb{R})$, cuspidal modular forms correspond to embeddings of discrete series representations into $L^{2}(\Gamma \backslash G)$, and cuspidal Maass forms to embeddings of unitary principal series representations. The realization of discrete series representations of $S L(2, \mathbb{R})$ as subrepresentations of principal series representations is very well known, making (3.6) quite concrete. For general groups, the Casselman embeddings cannot be described equally explicitly, nor do they need to be unique. Those are not obstacles to using $(3.6)$ in studying $L$-functions. In fact, the non-uniqueness is sometimes helpful in ruling out poles of $L$-functions.

Our tool in studying Rankin-Selberg and related $L$-functions is the pairing of automorphic distributions. In this paper, we shall only discuss RankinSelberg $L$-functions for $G L(2)$ and the exterior square $L$-function for $G L(4)$. Both involve the pairing of automorphic distributions of $G L(2)$. To minimize notational effort, we shall work with the group

$$
\begin{aligned}
G=P G L(2, \mathbb{R}) & \cong S L^{ \pm}(2, \mathbb{R}) /\{ \pm 1\} \\
& \left(S L^{ \pm}(2, \mathbb{R})=\{g \in G L(2, \mathbb{R}) \mid \operatorname{det} g= \pm 1\}\right),
\end{aligned}
$$

rather than $G=G L(2, \mathbb{R})$, for the remainder of this section. We let $B \subset G$ denote the lower triangular subgroup. For $\lambda \in \mathbb{C}$ and $\delta \in \mathbb{Z} / 2 \mathbb{Z}$, we define

$$
\chi_{\lambda, \delta}: B \rightarrow \mathbb{C}^{*}, \quad \chi_{\lambda, \delta}\left(\begin{array}{ll}
a & 0 \\
c & d
\end{array}\right)=\left(\operatorname{sgn} \frac{a}{d}\right)^{\delta}\left|\frac{a}{d}\right|^{\frac{\lambda}{2}} .
$$

The parameterization of the principal series involves a " $\rho$-shift", i.e., a shift by the half-sum of the positive roots. In our concrete setting

$$
\rho=1,
$$

and we shall write $\chi_{\lambda-\rho, \delta}$ instead of $\chi_{\lambda-1, \delta}$ to be consistent with the usual notation in the subject. The space of $C^{\infty}$ vectors for the principal series representation $\pi_{\lambda, \delta}$ is

$$
V_{\lambda, \delta}^{\infty}=\left\{F \in C^{\infty}(G) \mid F(g b)=\chi_{\lambda-\rho, \delta}\left(b^{-1}\right) F(g) \text { for all } g \in G, b \in B\right\},
$$

with action

$$
\left(\pi_{\lambda, \delta}(g) F\right)(h)=F\left(g^{-1} h\right) \quad\left(F \in V_{\lambda, \delta}^{\infty}, g, h \in G\right) .
$$

Quite analogously

$$
V_{\lambda, \delta}^{-\infty}=\left\{\tau \in C^{-\infty}(G) \mid \tau(g b)=\chi_{\lambda-\rho, \delta}\left(b^{-1}\right) \tau(g) \text { for all } g \in G, b \in B\right\}
$$


is the space of distribution vectors, on which $G$ acts by the same formula as on $V_{\lambda, \delta}^{\infty}$.

The tautological action of $G L(2, \mathbb{R})$ on $\mathbb{R}^{2}$ induces a transitive action of $G=P G L(2, \mathbb{R})$ on $\mathbb{R P}^{1}$; in fact $\mathbb{R P}^{1} \cong G / B$, since $B$ is the isotropy subgroup at the line spanned by the second standard basis vector of $\mathbb{R}^{2}$. According to the so-called "fundamental theorem of projective geometry", the action of $G$ on $\mathbb{R P}^{1}$ induces a simply transitive, faithful action on the set of triples of distinct points in $\mathbb{R} \mathbb{P}^{1} \times \mathbb{R} \mathbb{P}^{1} \times \mathbb{R} \mathbb{P}^{1}$. Put differently, $G$ has a dense open orbit in

$$
\mathbb{R} \mathbb{P}^{1} \times \mathbb{R} \mathbb{P}^{1} \times \mathbb{R} \mathbb{P}^{1} \cong G / B \times G / B \times G / B,
$$

and can be identified with that dense open orbit once a base point has been chosen. The three matrices

$$
f_{1}=\left(\begin{array}{ll}
1 & 0 \\
0 & 1
\end{array}\right), \quad f_{2}=\left(\begin{array}{ll}
1 & 1 \\
0 & 1
\end{array}\right), \quad f_{3}=\left(\begin{array}{cc}
0 & -1 \\
1 & 0
\end{array}\right)
$$

lie in distinct cosets of $B$, so

$$
G \hookrightarrow G / B \times G / B \times G / B, \quad g \mapsto\left(g f_{1} B, g f_{2} B, g f_{3} B\right)
$$

gives a concrete identification of $G$ with its open orbit in $\mathbb{R} \mathbb{P}^{1} \times \mathbb{R} \mathbb{P}^{1} \times \mathbb{R} \mathbb{P}^{1}$.

Formally at least, the existence of the open orbit can be used to define a $G$-invariant trilinear pairing

$$
\begin{aligned}
& V_{\lambda_{1}, \delta_{1}}^{\infty} \times V_{\lambda_{2}, \delta_{2}}^{\infty} \times V_{\lambda_{3}, \delta_{3}}^{\infty} \longrightarrow \mathbb{C}, \\
&\left(F_{1}, F_{2}, F_{3}\right) \mapsto P\left(F_{1}, F_{2}, F_{3}\right)={ }_{\operatorname{def}} \int_{G} F_{1}\left(g f_{1}\right) F_{2}\left(g f_{2}\right) F_{3}\left(g f_{3}\right) d g,
\end{aligned}
$$

between any three principal series representations. Although the $G$-invariance of the pairing is obvious from this formula, it is not clear that the integral converges. Before addressing the question of convergence, we should remark that the "fundamental theorem of projective geometry" is field-independent. The same ideas have been used to construct triple pairings for representations of $P G L\left(2, \mathbb{Q}_{p}\right)$. We should also point out that a different choice of base points $f_{j}$ would have the effect of multiplying the pairing by a non-zero constant.

The question of convergence of the integral (3.16) is most easily understood in terms of the "unbounded realization" of the principal series, which we discuss next. The subgroup

$$
N=\left\{n_{x}=\left(\begin{array}{ll}
1 & x \\
0 & 1
\end{array}\right) \mid x \in \mathbb{R}\right\} \cong \mathbb{R}
$$

of $G$ acts freely on $G / B$, and its image omits only a single point, the coset of

$$
s=\left(\begin{array}{cc}
0 & -1 \\
1 & 0
\end{array}\right) .
$$

It follows that any $F \in V_{\lambda, \delta}^{\infty}$ is completely determined by its restriction to $N \cong \mathbb{R}$; the defining identities 3.83 .10 imply that $\phi_{0}=$ restriction of $F$ to 
$\mathbb{R}$ is related to $\phi_{\infty}=$ restriction of $\pi_{\lambda, \delta}(s) F$ to $\mathbb{R}$ by the identity $\phi_{\infty}(x)=$ $|x|^{\lambda-1} \phi(-1 / x)$. This leads naturally to the identification

$$
V_{\lambda, \delta}^{\infty} \cong\left\{\left.\phi \in C^{\infty}(\mathbb{R})|| x\right|^{\lambda-1} \phi(-1 / x) \in C^{\infty}(\mathbb{R})\right\},
$$

with action

$$
\begin{array}{r}
\left(\pi_{\lambda, \delta}(g) \phi\right)(x)=(\operatorname{sgn}(a d-b c))^{\delta}\left(\frac{|c x+d|}{\sqrt{|a d-b c|}}\right)^{\lambda-1} \phi\left(\frac{a x+b}{c x+d}\right) \\
\text { for } g^{-1}=\left(\begin{array}{ll}
a & b \\
c & d
\end{array}\right) \in G .
\end{array}
$$

If $\left(\phi_{1}, \phi_{2}, \phi_{3}\right) \in\left(C^{\infty}(\mathbb{R})\right)^{3}$ correspond to $\left(F_{1}, F_{2}, F_{3}\right) \in V_{\lambda_{1}, \delta_{1}}^{\infty} \times V_{\lambda_{2}, \delta_{2}}^{\infty} \times V_{\lambda_{3}, \delta_{3}}^{\infty}$ via the unbounded realization 3.19,

$$
\begin{aligned}
& P\left(F_{1}, F_{2}, F_{3}\right)=\int_{\mathbb{R}^{3}} \phi_{1}(x) \phi_{2}(y) \phi_{3}(z) k(x, y, z) d x d y d z, \text { with } \\
& k(x, y, z)=\operatorname{sgn}((x-y)(y-z)(z-x))^{\delta_{1}+\delta_{2}+\delta_{3}} \times \\
& \times|x-y|^{\frac{-\lambda_{1}-\lambda_{2}+\lambda_{3}-1}{2}}|y-z|^{\frac{\lambda_{1}-\lambda_{2}-\lambda_{3}-1}{2}}|x-z|^{\frac{-\lambda_{1}+\lambda_{2}-\lambda_{3}-1}{2}} .
\end{aligned}
$$

This can be seen from the explicit form of the isomorphism (3.19), coupled with the definition 3.10 of $V_{\lambda, \delta}^{\infty}$. We should point out that in the setting of Maass forms, $\delta$ plays the role of the parity $\eta$ in 2.21.

Contrary to appearance, the integral (3.21) is really an integral over the compact space $\mathbb{R P}^{1} \times \mathbb{R} \mathbb{P}^{1} \times \mathbb{R P}^{1}$ : the integral retains the same general form when one or more of the coordinates $x, y, z$ are replaced by their reciprocals; this follows from the behavior of the $\phi_{j}$ at $\infty$ specified in $(3.19)$. The convergence of the integral is therefore a purely local matter. Near points where exactly two of the coordinates coincide, absolute convergence is guaranteed when the real part of the corresponding exponent is greater than -1 . To analyze the convergence near points of the triple diagonal $\{x=y=z\}$, it helps to "blow up" the triple diagonal in the sense of real algebraic geometry - or equivalently, to use polar coordinates in the normal directions. One then sees that absolute convergence requires not only the earlier condition

$$
\operatorname{Re}\left(\lambda_{i}-\lambda_{j}-\lambda_{k}\right)>-1 \quad \text { if } i \neq j, j \neq k, k \neq i,
$$

but also

$$
\operatorname{Re}\left(\lambda_{1}+\lambda_{2}+\lambda_{3}\right)<1 .
$$

Both conditions certainly hold when the $V_{\lambda_{i}, \delta_{i}}$ belong to the unitary principal series, i.e., when all the $\lambda_{j}$ are purely imaginary.

The argument we have sketched establishes the existence of an invariant trilinear pairing between the spaces of $C^{\infty}$ vectors of any three unitary principal series representations. The pairing is known to be unique up to scaling [16]. Even when the $\lambda_{i}$ are not purely imaginary, one can use (3.21) to exhibit an invariant trilinear pairing by meromorphic continuation. Indeed, for compactly 
supported functions of one variable, the functional $f \mapsto \int_{\mathbb{R}} f(x)|x|^{s-1} d x$ extends meromorphically to $s \in \mathbb{C}$, with first order poles at the non-positive integers, but no other poles. As was just argued, the integral kernel in (3.21) can be expressed as $|u|^{s}$ or $|u|^{s_{1}}|v|^{s_{2}}$, in terms of suitable local coordinates, after blowing up when necessary. Localizing the problem as before, by means of a suitable partition of unity, one can therefore assign a meaning to the integral (3.21) for all triples $\left(\lambda_{1}, \lambda_{2}, \lambda_{3}\right) \in \mathbb{C}^{3}$ outside certain hyperplanes, where the integral has poles. Even for parameters $\left(\lambda_{1}, \lambda_{2}, \lambda_{3}\right)$ on these hyperplanes one can exhibit an invariant triple pairing by taking residues.

Let us now consider the datum of distribution vectors $\tau_{j} \in V_{\lambda_{j}, \delta_{j}}^{-\infty}$ for three principal series representations $V_{\lambda_{j}, \delta_{j}}, 1 \leq j \leq 3$. The unbounded realization of the $V_{\lambda_{j}, \delta_{j}}^{-\infty}$ is slightly more complicated than the $C^{\infty}$ case 3.19 : unlike a $C^{\infty}$ function, a distribution is not determined by its restriction to a dense open subset of its domain. The distribution analogue of (3.19),

$$
V_{\lambda, \delta}^{-\infty} \cong\left\{\left.\left(\sigma_{0}, \sigma_{\infty}\right) \in\left(C^{-\infty}(\mathbb{R})\right)^{2}\left|\sigma_{\infty}(x)=\right| x\right|^{\lambda-1} \sigma_{0}(-1 / x)\right\},
$$

therefore involves a pair of distributions on $\mathbb{R}$ that determine each other on $\mathbb{R}-\{0\}$. Suppose now that $\tau_{j} \cong\left(\sigma_{j, 0}, \sigma_{j, \infty}\right)$ via 3.24. Then

$$
\begin{aligned}
(x, y, z) & \mapsto \sigma_{1,0}(x) \sigma_{2,0}(y) \sigma_{3,0}(z) \operatorname{sgn}((x-y)(y-z)(z-x))^{\delta_{1}+\delta_{2}+\delta_{3}} \times \\
& \times|x-y|^{\frac{-\lambda_{1}-\lambda_{2}+\lambda_{3}+1}{2}}|y-z|^{\frac{\lambda_{1}-\lambda_{2}-\lambda_{3}+1}{2}}|x-z|^{\frac{-\lambda_{1}+\lambda_{2}-\lambda_{3}+1}{2}}
\end{aligned}
$$

extends naturally to a distribution on $\left\{(x, y, z) \in\left(\mathbb{R P}^{1}\right)^{3} \mid x \neq y \neq z \neq x\right\}$; as one or more of the coordinates tend to $\infty$, one replaces those coordinates by the negative of their reciprocals, and simultaneously the corresponding $\sigma_{j, 0}$ by $\sigma_{j, \infty}$. Since $\left\{(x, y, z) \in\left(\mathbb{R P}^{1}\right)^{3} \mid x \neq y \neq z \neq x\right\} \cong G$ via the identification $(3.15)$, we may regard (3.25) as a distribution on $G$. In fact, this distribution is

$$
\left\{g \mapsto \tau_{1}\left(g f_{1}\right) \tau_{2}\left(g f_{2}\right) \tau_{3}\left(g f_{3}\right)\right\} \in C^{-\infty}(G),
$$

although the latter description has no immediately obvious meaning without the steps we have just gone through. The apparent discrepancy between the signs in the exponents in (3.21) and 3.25) reflects the fact that

$$
|x-y|^{-1}|y-z|^{-1}|z-x|^{-1} \cong d g=\text { Haar measure on } G
$$

via the identification 3.15 . Let us formally record the substance of our discussion:

3.28 Observation. For $\tau_{j} \in V_{\lambda_{j}, \delta_{j}}^{-\infty}, 1 \leq j \leq 3$,

$$
g \mapsto \tau_{1}\left(g f_{1}\right) \tau_{2}\left(g f_{2}\right) \tau_{3}\left(g f_{3}\right)
$$

is a well defined distribution on $G$. 
To motivate our result on pairing of automorphic distributions, we temporarily deviate from our standing assumption that $\Gamma \subset G$ be arithmetically defined; instead we suppose that $\Gamma \subset G$ is a discrete, cocompact subgroup. In that case, if $\tau_{j} \in\left(V_{\lambda_{j}, \delta_{j}}^{-\infty}\right)^{\Gamma}, 1 \leq j \leq 3$, are $\Gamma$-invariant distribution vectors, (3.26) defines a distribution on the compact manifold $\Gamma \backslash G$. As such, it can be integrated against the constant function 1 , and

$$
\int_{\Gamma \backslash G} \tau_{1}\left(g f_{1}\right) \tau_{2}\left(g f_{2}\right) \tau_{3}\left(g f_{3}\right) d g
$$

has definite meaning. The value of the integral remains unchanged when the variable of integration $g$ is replaced by $g h$, for any particular $h \in G$. Thus, if $\psi \in C_{c}^{\infty}(G)$ has total integral one,

$$
\begin{aligned}
\int_{\Gamma \backslash G} & \tau_{1}\left(g f_{1}\right) \tau_{2}\left(g f_{2}\right) \tau_{3}\left(g f_{3}\right) d g= \\
= & \int_{G} \int_{\Gamma \backslash G} \tau_{1}\left(g h f_{1}\right) \tau_{2}\left(g h f_{2}\right) \tau_{3}\left(g h f_{3}\right) \psi(h) d g d h \\
= & \int_{\Gamma \backslash G}\left(\int_{G} \tau_{1}\left(g h f_{1}\right) \tau_{2}\left(g h f_{2}\right) \tau_{3}\left(g h f_{3}\right) \psi(h) d h\right) d g .
\end{aligned}
$$

The implicit use of Fubini's theorem at the second step can be justified by a partition of unity argument. In short, we have expressed the integral (3.29) as the integral over $\Gamma \backslash G$ of the $\Gamma$-invariant function

$$
g \mapsto \int_{G} \tau_{1}\left(g h f_{1}\right) \tau_{2}\left(g h f_{2}\right) \tau_{3}\left(g h f_{3}\right) \psi(h) d h .
$$

This function is smooth, like any convolution of a distribution with a compactly supported test function. Note that the integral (3.31) is well defined even for parameters $\left(\lambda_{1}, \lambda_{2}, \lambda_{3}\right) \in \mathbb{C}^{3}$ which correspond to poles of the integral (3.21).

We now return to our earlier setting, of an arithmetically defined subgroup $\Gamma \subset G=P G L(2, \mathbb{R})$, specifically a congruence subgroup

$$
\Gamma \subset P G L(2, \mathbb{Z}) .
$$

In this context, the integral 3.29 has no obvious meaning, since we would have to integrate a distribution over the noncompact manifold $\Gamma \backslash G$. The "smoothed" integral (3.30), however, potentially makes sense: if the integrand (3.31) can be shown to decay rapidly towards the cusps of $\Gamma \backslash G$, it is simply an ordinary, convergent integral. That is the case, under appropriate hypotheses:

3.33 Theorem. Let $\tau_{j} \in\left(V_{\lambda_{j}, \delta_{j}}^{-\infty}\right)^{\Gamma}, 1 \leq j \leq 3$, be $\Gamma$-automorphic distributions, and $\psi \in C_{c}^{\infty}(G)$ a test function, subject to the normalizing condition

$$
\int_{G} \psi(g) d g=1
$$


If at least one of the $\tau_{j}$ is cuspidal, the $\Gamma$-invariant $C^{\infty}$ function

$$
F(g)=\int_{G} \tau_{1}\left(g h f_{1}\right) \tau_{2}\left(g h f_{2}\right) \tau_{3}\left(g h f_{3}\right) \psi(h) d h
$$

decays rapidly along the cusps of $\Gamma$; in particular $\int_{\Gamma \backslash G} F(g) d g$ converges absolutely. This integral does not depend on the specific choice of $\psi$. If, in addition, one of the $\tau_{j}$ depends holomorphically on a complex parameter,

$$
\int_{\Gamma \backslash G} F(g) d g=\int_{\Gamma \backslash G} \int_{G} \tau_{1}\left(g h f_{1}\right) \tau_{2}\left(g h f_{2}\right) \tau_{3}\left(g h f_{3}\right) \psi(h) d h d g
$$

also depends holomorphically on that parameter.

Why does $F$ decay rapidly? It is not a modular form - the Casimir operator of $G$ does not act on it finitely. Nor does $F$ satisfy the condition of cuspidality. However, $F$ can be expressed as the restriction to the diagonal of a modular form in three variables:

$$
\left(g_{1}, g_{2}, g_{3}\right) \mapsto \int_{G} \tau_{1}\left(g_{1} h f_{1}\right) \tau_{2}\left(g_{2} h f_{2}\right) \tau_{3}\left(g_{3} h f_{3}\right) \psi(h) d h
$$

is a $C^{\infty}$ function on $G \times G \times G$; this follows from the fact that the cosets $f_{j} B$ lie in general position. Since $\tau_{j} \in\left(V_{\lambda_{j}, \delta_{j}}^{-\infty}\right)^{\Gamma},(3.34)$ is a $\Gamma$-invariant eigenfunction of the Casimir operator in each of the variables separately. It is cuspidal in the variable corresponding to the cuspidal factor $\tau_{j}$, hence decays rapidly in this one direction. It has at worst moderate growth in the other directions, and therefore decays rapidly when restricted to the diagonal. The remaining assertions of the lemma are relatively straightforward.

We shall need a variant of the theorem in the last section, for the analysis of the exterior square $L$-function for $G L(4)$. Two of the $\tau_{j}$ then occur coupled, as a distribution vector for a principal series representation of $G \times G, \Gamma$-invariant only under the diagonal action, not separately. These two $\tau_{j}$ arise from a single cuspidal automorphic distribution $\tau$ for $G L(4, \mathbb{R})$. In this situation the rapid decay of $F$ reflects the cuspidality of $\tau$.

\section{The Rankin-Selberg $L$-function for $G L(2)$}

The argument we are about to sketch parallels the classical arguments of Rankin 17] and Selberg [18, and of Jacquet [6] in the case of Maass forms. We shall pair two automorphic distributions against an Eisenstein series. In our setting, of course, the Eisenstein series is also an automorphic distribution.

We recall the construction of the distribution Eisenstein series from 15, specialized to the case of $G=P G L(2, \mathbb{R})$. To simplify the discussion, we only work at full level - in other words, with 


$$
\Gamma=P G L(2, \mathbb{Z}) \simeq S L^{ \pm}(2, \mathbb{Z}) /\{ \pm 1\} .
$$

We define $\delta_{\infty} \in V_{\nu, 0}^{-\infty}$ in terms of the unbounded realization 3.24): $\delta_{\infty}$ corresponds to $\left(\sigma_{0}, \sigma_{\infty}\right)$, with $\sigma_{0}=0$ and $\sigma_{\infty}=$ Dirac delta function at 0 . Then $\pi_{\nu, 0}(\gamma) \delta_{\infty}=\delta_{\infty}$ for all $\gamma \in \Gamma_{\infty}=\{\gamma \in \Gamma \mid \gamma \infty=\infty\}$. In particular, the series

$$
E_{\nu} \in V_{\nu, 0}^{-\infty}, \quad E_{\nu}=\zeta(\nu+1) \sum_{\gamma \in \Gamma / \Gamma_{\infty}} \pi_{\nu, 0}(\gamma) \delta_{\infty},
$$

makes sense at least formally. It is $\Gamma$-invariant by construction. Hence, when we describe $E_{\nu}$ in terms of the unbounded realization (3.24), it suffices to specify the first member $\sigma_{0}$ of the pair $\left(\sigma_{0}, \sigma_{\infty}\right)$. This allows us to regard $E_{\nu}$ as a distribution on the real line,

$$
E_{\nu} \cong \sum_{p, q \in \mathbb{Z}, q>0} q^{-\nu-1} \delta_{p / q}(x) .
$$

To see the equivalence of 4.2 and 4.3 , we note that $\delta_{p / q}(x)$, with $p, q \in \mathbb{Z}$ relatively prime, corresponds to the translate of $\delta_{\infty}$ under

$$
\left(\begin{array}{cc}
p & r \\
q & s
\end{array}\right) \in \Gamma, \quad \text { with } r, s \in \mathbb{Z} \text { chosen so that } s p-r q=1 .
$$

The disappearance of the factor $\zeta(\nu+1)$ in 4.3$)$ reflects the fact that we now sum over all pairs of integers $p, q$, with $q>0$, not over relatively prime pairs.

The integral of the series 4.3 against a compactly supported test function converges uniformly and absolutely when $\operatorname{Re} \nu>1$. Hence $E_{\nu} \in V_{\nu, 0}^{-\infty}$ is well defined for $\operatorname{Re} \nu>1$, and depends holomorphically on $\nu$ in this region. The periodic distribution 4.3 has a Fourier expansion,

$$
E_{\nu} \simeq \sum_{n \in \mathbb{Z}} a_{n} e(n x) .
$$

To calculate the Fourier coefficients, we reinterpret the sum as a distribution on $\mathbb{R} / \mathbb{Z}$. Then

$$
\begin{aligned}
a_{n} & =\int_{\mathbb{R} / \mathbb{Z}} e(-n x) \sum_{p, q \in \mathbb{Z}, q>0} q^{-\nu-1} \delta_{p / q}(x) d x \\
& =\sum_{q>0} \sum_{0 \leq p<q} q^{-\nu-1} e(-n p / q)= \begin{cases}\sum_{d \mid n} d^{-\nu} & \text { if } n \neq 0 \\
\zeta(\nu) & \text { if } n=0 .\end{cases}
\end{aligned}
$$

The $a_{n}, n \neq 0$, are entire functions of $\nu$, whereas $a_{0}=\zeta(\nu)$ has a pole at $\nu=1$, so

$$
\begin{gathered}
E_{\nu} \text { extends meromorphically to the entire complex plane, } \\
\text { with a single pole at } \nu=1 \text {, of order one. }
\end{gathered}
$$

We should remark that $\delta_{\infty}$ is even with respect to the involution $x \mapsto-x$. This is the reason why at full level there is no Eisenstein series of odd parity i.e., no Eisenstein series in $V_{\nu, 1}^{-\infty}$. 
The Eisenstein series 4.2 satisfies a functional equation, which relates $E_{-\nu} \in V_{-\nu, 0}^{-\infty}$ to $E_{\nu} \in V_{\nu, 0}^{-\infty}$ via the intertwining operator

$$
J_{\nu}: V_{-\nu, 0}^{-\infty} \longrightarrow V_{\nu, 0}^{-\infty}
$$

On the level of $C^{\infty}$ vectors, and in terms of the unbounded realization 3.19, the operator is given by the formula

$$
\left(J_{\nu} \phi\right)(x)=\int_{\mathbb{R}} \phi(y)|y-x|^{\nu-1} d y .
$$

Because of the condition on $\phi$ at infinity, this integral has no singularity at $y=\infty$. At $y=x$, the integral converges when $\operatorname{Re} \nu>0$, but continues meromorphically to the entire complex plane. It is known that the integral transform 4.9 extends continuously from an operator $J_{\nu}: V_{-\nu, 0}^{\infty} \rightarrow V_{\nu, 0}^{\infty}$ between the spaces of $C^{\infty}$ vectors to the operator (4.8). Alternatively and equivalently, (4.8) can be defined as the adjoint of $J_{\nu}: V_{-\nu, 0}^{\infty} \rightarrow V_{\nu, 0}^{\infty}$, using the natural duality ${ }^{3}$ between $V_{\nu, 0}^{\infty}$ and $V_{-\nu, 0}^{-\infty}$. Either way one sees that

$$
V_{-\nu, 0}^{-\infty} \ni e(n x) \stackrel{J_{\nu}}{\longrightarrow} G_{0}(\nu)|n|^{-\nu} e(n x) \in V_{\nu, 0}^{-\infty} \quad(n \neq 0) .
$$

Here $G_{0}(\nu)$ refers to the Gamma factor described in (2.25), and $e(n x)$ is shorthand for the pair $\left(e(n x),|x|^{\mp \nu-1} e(-n / x)\right)-\mathrm{cf}$. (3.24); the second member of the pair can be given a definite meaning even at the origin, using the notion of vanishing to infinite order that was discussed in section 2

In view of the relation (4.10), $J_{\nu}$ maps the Fourier series (4.6) for $E_{-\nu}$ to $G_{0}(\nu)$ times the corresponding series for $E_{\nu}$, except possibly for the constant term and a distribution supported at infinity. However, no non-zero linear combination of a constant function and a distribution supported at infinity can be $\Gamma$-invariant. This proves

$$
J_{\nu} E_{-\nu}=G_{0}(\nu) E_{\nu} .
$$

That is the functional equation satisfied by the Eisenstein series. The parameter $\nu$ is natural from the point of view of representation theory. In the eventual application, we shall work with

$$
s=(\nu+1) / 2
$$

instead. Note that $\nu \mapsto-\nu$ corresponds to $s \mapsto 1-s$.

We now fix two automorphic distributions, either of which may arise from a modular form or a Maass form,

$$
\tau_{1} \in\left(V_{\lambda_{1}, \delta_{1}}^{-\infty}\right)^{\Gamma} \text { and } \tau_{2} \in\left(V_{\lambda_{2}, \delta_{2}}^{-\infty}\right)^{\Gamma},
$$

\footnotetext{
${ }^{3}$ The duality which extends the $G$-invariant pairing $V_{\nu, 0}^{\infty} \times V_{-\nu, 0}^{\infty} \rightarrow \mathbb{C}$ given by integration over $\mathbb{R}$, in terms of the unbounded realization.
} 
of which at least one is cuspidal. According to 4.7) and theorem 3.33, the integral

$$
P_{\nu}^{\Gamma}\left(\tau_{1}, \tau_{2}, E_{\nu}\right)=\int_{\Gamma \backslash G} \int_{G} \tau_{1}\left(g h f_{1}\right) \tau_{2}\left(g h f_{2}\right) E_{\nu}\left(g h f_{3}\right) \psi(h) d h d g
$$

depends meromorphically on $\nu \in \mathbb{C}$, with a potential first order pole at $\nu=1$ but no other singularities. The subscript $\nu$ is meant to emphasize the fact that the third argument lies in the space $\left(V_{\nu, 0}^{-\infty}\right)^{\Gamma}$, and the superscript $\Gamma$ distinguishes this pairing of $\Gamma$-invariant distribution vectors from the pairing (3.21) between spaces of $C^{\infty}$ vectors.

We shall derive the Rankin-Selberg functional equation from the functional equation (4.11) of the Eisenstein series. Since the latter involves the intertwining operator, we need to know how $J_{\nu}$ relates $P_{-\nu}^{\Gamma}$ to $P_{\nu}^{\Gamma}$. First the analogous statement about the pairing (3.21]: for $F_{1} \in V_{\lambda_{1}, \delta_{1}}^{\infty}, F_{2} \in V_{\lambda_{2}, \delta_{2}}^{\infty}, F_{3} \in V_{-\nu, 0}^{\infty}$,

$$
\begin{aligned}
& P\left(F_{1}, F_{2}, J_{\nu} F_{3}\right)= \\
& =(-1)^{\delta_{1}+\delta_{2}} \frac{G_{\delta_{1}+\delta_{2}}\left(\frac{\lambda_{1}-\lambda_{2}-\nu+1}{2}\right) G_{\delta_{1}+\delta_{2}}\left(\frac{-\lambda_{1}+\lambda_{2}-\nu+1}{2}\right)}{G_{0}(1-\nu)} P\left(F_{1}, F_{2}, F_{3}\right) .
\end{aligned}
$$

Note that $P(\ldots)$ on the left and the right side of the equality refer to the pairing $V_{\lambda_{1}, \delta_{1}}^{\infty} \times V_{\lambda_{2}, \delta_{2}}^{\infty} \times V_{\nu, 0}^{\infty} \rightarrow \mathbb{C}$, respectively $V_{\lambda_{1}, \delta_{1}}^{\infty} \times V_{\lambda_{2}, \delta_{2}}^{\infty} \times V_{-\nu, 0}^{\infty} \rightarrow \mathbb{C}$. The Gamma factors $G_{\delta}(\ldots)$ have the same meaning as in (2.25). Since both sides of the equality depend meromorphically on $\nu$, it suffices to establish it for values of $\nu$ in some non-empty open region. In view of (3.21) and 4.9), the assertion 4.15 reduces to the identity

$$
\begin{aligned}
& \int_{\mathbb{R}} \frac{(\operatorname{sgn}(y-t)(t-x))^{\delta_{1}+\delta_{2}}}{(\operatorname{sgn}(y-z)(z-x))^{\delta_{1}+\delta_{2}}}|x-t|^{\alpha-1}|y-t|^{\beta-1}|z-t|^{-\alpha-\beta} d t= \\
& \quad=(-1)^{\delta_{1}+\delta_{2}} \frac{G_{\delta_{1}+\delta_{2}}(\alpha) G_{\delta_{1}+\delta_{2}}(\beta)}{G_{0}(\alpha+\beta)}|x-y|^{\alpha+\beta-1}|x-z|^{-\beta}|y-z|^{-\alpha},
\end{aligned}
$$

with $\alpha=\left(-\lambda_{1}+\lambda_{2}-\nu+1\right) / 2, \beta=\left(\lambda_{1}-\lambda_{2}-\nu+1\right) / 2$. The integral converges in the region $\operatorname{Re} \alpha>0, \operatorname{Re} \beta>0, \operatorname{Re}(\alpha+\beta)<1$. The uniqueness of the triple pairing ensures that 4.15 must be correct up to a multiplicative constant. But then (4.16) must also be correct, except possibly for the specific constant of proportionality. That constant can be pinned down in a variety of ways; see, for example, [15, Lemma 4.32].

A partition of unity argument shows that the quantities $P_{\nu}^{\Gamma}\left(\tau_{1}, \tau_{2}, J_{\nu} E_{-\nu}\right)$ and $P_{-\nu}^{\Gamma}\left(\tau_{1}, \tau_{2}, E_{-\nu}\right)$ are related by the same Gamma factors as the global pairings in 4.15. Combining this information with 4.11) and the standard Gamma identity $G_{\delta}(\nu) G_{\delta}(1-\nu)=(-1)^{\delta}$, we find

$$
\begin{aligned}
& P_{\nu}^{\Gamma}\left(\tau_{1}, \tau_{2}, E_{\nu}\right)= \\
& \quad=(-1)^{\delta_{1}+\delta_{2}} G_{\delta_{1}+\delta_{2}}\left(\frac{\lambda_{1}-\lambda_{2}-\nu+1}{2}\right) G_{\delta_{1}+\delta_{2}}\left(\frac{-\lambda_{1}+\lambda_{2}-\nu+1}{2}\right) P_{-\nu}^{\Gamma}\left(\tau_{1}, \tau_{2}, E_{-\nu}\right) .
\end{aligned}
$$


Once we relate $P_{\nu}^{\Gamma}\left(\tau_{1}, \tau_{2}, E_{\nu}\right)$ to the Rankin-Selberg $L$-function, this identity will turn out be the functional equation.

We begin by substituting the expression 4.2 for $E_{\nu}$ in (4.14). Initially we argue formally; the unfolding step will be justified later, when we see that the resulting integral converges absolutely:

$$
\begin{aligned}
& P_{\nu}^{\Gamma}\left(\tau_{1}, \tau_{2}, E_{\nu}\right)=\int_{\Gamma \backslash G} \int_{G} \tau_{1}\left(g h f_{1}\right) \tau_{2}\left(g h f_{2}\right) E_{\nu}\left(g h f_{3}\right) \psi(h) d h d g \\
& \quad=\zeta(\nu+1) \sum_{\Gamma / \Gamma_{\infty}} \int_{\Gamma \backslash G} \int_{G} \tau_{1}\left(g h f_{1}\right) \tau_{2}\left(g h f_{2}\right) \delta_{\infty}\left(\gamma^{-1} g h f_{3}\right) \psi(h) d h d g \\
& \quad=\zeta(\nu+1) \int_{\Gamma_{\infty} \backslash G} \int_{G} \tau_{1}\left(g h f_{1}\right) \tau_{2}\left(g h f_{2}\right) \delta_{\infty}\left(g h f_{3}\right) \psi(h) d h d g .
\end{aligned}
$$

The integrand for the outer integral on the right is no longer $\Gamma$-invariant, but it is $(\Gamma \cap N)$-invariant, of course, and has all the other properties of the integrand in (4.14). Those are the properties used in the proof of theorem 3.33 to establish rapid decay. In other words, the same argument shows that the integrand in (4.18) decays rapidly in the direction of the cusp. However, $\Gamma_{\infty} \backslash G$ is not "compact in the directions opposite to the cusp", and we still need to argue that the integral converges in those directions as well.

Together with the upper triangular unipotent subgroup $N \subset G$, the two subgroups

$$
K=S O(2) /\{ \pm 1\}, \quad A=\left\{a_{t}=\left(\begin{array}{cc}
e^{t} & 0 \\
0 & e^{-t}
\end{array}\right) \mid t \in \mathbb{R}\right\}
$$

determine the Iwasawa decomposition

$$
G^{0}=N A K
$$

of the identity component $G^{0} \simeq S L(2, \mathbb{R}) /\{ \pm 1\}$ of $G$. Since $\Gamma_{\infty}$ meets both components of $G$, and since $\Gamma_{\infty} \cap G^{0}=\Gamma \cap N$, we can make the identification $\Gamma_{\infty} \backslash G \simeq(\Gamma \cap N) \backslash G^{0}$. Hence, and because

$$
d g=e^{-2 \rho}(a) d n d a d k, \quad \text { with } e^{\rho}\left(a_{t}\right)=e^{t},
$$

the identity 4.18 can be rewritten as

$$
\begin{aligned}
& P_{\nu}^{\Gamma}\left(\tau_{1}, \tau_{2}, E_{\nu}\right)= \\
& =\zeta(\nu+1) \int_{K} \int_{A} \int_{(\Gamma \cap N) \backslash N} \int_{G} e^{-2 \rho}(a) \tau_{1}\left(n a k h f_{1}\right) \tau_{2}\left(n a k h f_{2}\right) \times \\
& \times \delta_{\infty}\left(n a k h f_{3}\right) \psi(h) d h d n d a d k .
\end{aligned}
$$

As the $t$ tends to $+\infty$, the point $g=n a_{t} k$ moves towards the cusp. In the opposite direction, as $t \rightarrow-\infty$, the integrand in 4.22 grows at most like a power of $e^{-t}$. To see this, and to determine the rate of growth or decay, we 
temporarily regard the three instances of the argument nak as independent of each other, as in the discussion around (3.34). In the case of the $\tau_{j}$, the maximum rate of growth is $e^{\left(-\left|\operatorname{Re} \lambda_{j}\right|+1\right) t}$, and in the case of $\delta_{\infty}$, it is $e^{(\operatorname{Re} \nu+1) t}$, without absolute value sign around Re $\nu$. The reason for the latter assertion is that we know the behavior of $\delta_{\infty}(g)$ when $g$ is multiplied on the left by any $n \in N$ - unchanged - and when $g$ is multiplied on the left by any $a_{t} \in A$ - by the factor $e^{(\operatorname{Re} \nu+1) t}$; cf. 4.28$)$ below. In short, the integrand in 4.22 can be made to decay as $t \rightarrow-\infty$ by choosing Re $\nu$ large enough. That makes the integral converge absolutely and justifies the unfolding process.

The smoothing function $\psi \in C_{c}^{\infty}(G)$ in theorem 3.33 is arbitrary so far, except for the normalization $\int_{G} \psi(g) d g=1$. We can therefore require $\psi$ to have support in $G^{0}$, and also impose the condition

$$
\psi(k g)=\psi(g) \text { for all } k \in K, g \in G ;
$$

the latter can be arranged by averaging the original function $\psi$ over $K$. The analogue of 4.21 for the $K A N$ decomposition is $d g=e^{2 \rho}(a) d k d a d n$. Hence

$$
\begin{array}{r}
\int_{A} \int_{N} e^{2 \rho}(a) \psi(a n) d n d a=1, \text { or equivalently } \int_{A} \psi_{A}(a) d a=1, \\
\text { with } \psi_{A}(a)=e^{2 \rho}(a) \int_{N} \psi(a n) d n=\int_{N} \psi(n a) d n,
\end{array}
$$

restates the normalization condition for the $K$-invariant function $\psi$.

We had argued earlier that the function $e(\ell x)$, for $\ell \neq 0$, has a canonical extension - now viewed as distribution - across infinity. That allows us to regard $e(\ell x)$ as a well defined element of the unbounded model (3.19). If $\operatorname{Re} \lambda<2$ - which will be the case for automorphic distributions corresponding to modular forms or to Maass forms - we can also make sense of the constant function 1 as element of the unbounded mode ${ }^{4}$. Whether or not $\ell$ equals zero, we let $B_{\ell, \lambda, \delta} \in V_{\lambda, \delta}^{-\infty}$ denote the distribution vector that corresponds to $e(\ell x)$. Then

$$
\pi_{\lambda, \delta}\left(n_{x}\right) B_{\ell, \lambda, \delta}=e(-\ell x) B_{\ell, \lambda, \delta}, \quad \text { and } \quad B_{\ell, \lambda, \delta}\left(n_{x}\right)=e(\ell x) .
$$

The latter equation has meaning since $N \subset G / B$ is open and $B_{\ell, \lambda, \delta}$, like any vector in $V_{\lambda, \delta}^{-\infty}$, transforms according to a character under right translation by elements of $B$. We had assumed that at least one among $\tau_{1}$ and $\tau_{2}$ is cuspidal $\tau_{1}$, say, for definiteness. Then

$$
\tau_{1}=\sum_{\ell \neq 0} a_{\ell} B_{\ell, \lambda_{1}, \delta_{1}}, \quad \tau_{2}=\sum_{\ell \in \mathbb{Z}} b_{\ell} B_{\ell, \lambda_{2}, \delta_{2}}+\ldots
$$

are the Fourier expansions of $\tau_{1}$ and $\tau_{2}$. Here $\ldots$ stands for a vector in $V_{\lambda_{2}, \delta_{2}}^{-\infty}$ that is $N$-invariant and supported on $s B \subset G / B$; recall (3.18) for the definition of $s \in G$. The series for $\tau_{1}$ has no such singular contribution on $s B$, as was explained in (2.17) and the passage that follows it.

\footnotetext{
${ }^{4}$ One can do so also for other values of $\lambda \in \mathbb{C}$, by meromorphic continuation.
} 
In 4.22 , the process of averaging over $\Gamma \backslash \Gamma_{\infty}$ from the left and smoothing from the right commute. Thus, using the fact that $\delta_{\infty}$ and ... in (4.26) are $N$-invariant, we find

$$
\begin{aligned}
& \int_{(\Gamma \cap N) \backslash N} \int_{G} \tau_{1}\left(n a k h f_{1}\right) \tau_{2}\left(n a k h f_{2}\right) \delta_{\infty}\left(n a k h f_{3}\right) \psi(h) d h d n= \\
& =\sum_{\ell \neq 0} a_{\ell} b_{-\ell} \int_{G} B_{\ell, \lambda_{1}, \delta_{1}}\left(a k h f_{1}\right) B_{-\ell, \lambda_{2}, \delta_{2}}\left(a k h f_{2}\right) \delta_{\infty}\left(a k h f_{3}\right) \psi(h) d h \\
& =\sum_{\ell \neq 0} a_{\ell} b_{-\ell} \int_{G} B_{\ell, \lambda_{1}, \delta_{1}}\left(a h f_{1}\right) B_{-\ell, \lambda_{2}, \delta_{2}}\left(a h f_{2}\right) \delta_{\infty}\left(a h f_{3}\right) \psi(h) d h \\
& =\sum_{\ell \neq 0} a_{\ell} b_{-\ell} \int_{G} B_{\ell, \lambda_{1}, \delta_{1}}(a h) B_{-\ell, \lambda_{2}, \delta_{2}}\left(a h n_{1}\right) \delta_{\infty}(a h s) \psi(h) d h ;
\end{aligned}
$$

at the second step we have used the $K$-invariance of $\psi$, and at the last step, we have inserted the concrete values $f_{1}=e, f_{2}=n_{1}, f_{3}=s-\mathrm{cf}$. (3.14) and (3.17).

When we substitute 4.27 into 4.22 , we can make several simplifications. The expression on the right in (4.27) no longer depends on the variable $k$, so the integral over $K$ in 4.22 can be omitted. The distribution $\delta_{\infty}$ is supported on $s B \subset G$. Hence, when the variable $h$ in $(4.22)$ is written as $h=k n \tilde{a}$, with $k \in K, n \in N, \tilde{a} \in A$, and $d h=d k d n d \tilde{a}$, the $k$-integration reduces to evaluation at $k=e$. Since $A$ acts via $e^{2 \rho}$ on the cotangent space at $s B \in G / B$,

$$
\delta_{\infty}(a k s) d k=e^{2 \rho}(a) \delta_{\infty}\left(k s a^{-1}\right) d k=\chi_{\nu+\rho}(a) \delta_{\infty}(k s) d k \text { for } a \in A .
$$

It follows that $\delta_{\infty}(a h s) d k=\delta_{\infty}(a k n \tilde{a} s) d k=\delta_{\infty}\left(a k s\left(s^{-1} n s\right) \tilde{a}^{-1}\right) d k$ contributes the factor $\chi_{\nu+\rho}(a) \chi_{\nu-\rho}(\tilde{a})=e^{-2 \rho}(\tilde{a}) \chi_{\nu+\rho}(a \tilde{a})$ when it is integrated over $K$. Effectively we have replaced the integrals over $h \in G$ in $(4.22)$ and (4.27) by integrals over $A N$. But the integrand being smoothed in (4.27) is already $N$-invariant. Thus, instead of smoothing over $G$ with respect to $\psi$, we only need to smooth over $A$ with respect to $\psi_{A}$, as defined in (4.24):

$$
\begin{aligned}
P_{\nu}^{\Gamma}\left(\tau_{1}, \tau_{2}, E_{\nu}\right) & =\zeta(\nu+1) \sum_{\ell \neq 0} a_{\ell} b_{-\ell} \int_{A} \int_{A} e^{-2 \rho}(a \tilde{a}) \times \\
& \times B_{\ell, \lambda_{1}, \delta_{1}}(a \tilde{a}) B_{-\ell, \lambda_{2}, d_{2}}\left(a \tilde{a} n_{1}\right) \chi_{\nu+\rho}(a \tilde{a}) \psi_{A}(\tilde{a}) d \tilde{a} d a .
\end{aligned}
$$

We parametrize $a, \tilde{a} \in A$ as $a=a_{t}, \tilde{a}=a_{\tilde{t}}$, as in 4.19 , with $t, \tilde{t} \in \mathbb{R}$ and $d a=d t, d \tilde{a}=d \tilde{t}$. Then, in view of the definition (3.12) of $V_{\lambda, \delta}^{-\infty}$ and the characterization 4.25 of $B_{\ell, \lambda, \delta}$,

$$
\begin{gathered}
B_{\ell, \lambda_{1}, \delta_{1}}\left(a_{t} a_{\tilde{t}}\right)=e^{\left(1-\lambda_{1}\right)(t+\tilde{t})} B_{\ell, \lambda_{1}, \delta_{1}}(e)=e^{\left(1-\lambda_{1}\right)(t+\tilde{t})}, \\
B_{-\ell, \lambda_{2}, \delta_{2}}\left(a_{t} \tilde{a}_{\tilde{t}} n_{1}\right)=e^{\left(1-\lambda_{2}\right)(t+\tilde{t})} B_{-\ell, \lambda_{2}, \delta_{2}}\left(a \tilde{a} n_{1} a^{-1} \tilde{a}^{-1}\right) \\
=e^{\left(1-\lambda_{2}\right)(t+\tilde{t})} e\left(-\ell e^{2(t+\tilde{t})}\right), \\
\chi_{\nu+\rho}\left(a_{t} a_{\tilde{t}}\right)=e^{(\nu+1)(t+\tilde{t})}, \quad e^{-2 \rho}\left(a_{t} a_{\tilde{t}}\right)=e^{-2(t+\tilde{t})} .
\end{gathered}
$$


This leads to the equation

$$
\begin{aligned}
P_{\nu}^{\Gamma}\left(\tau_{1}, \tau_{2}, E_{\nu}\right) & =\zeta(\nu+1) \sum_{\ell \neq 0} a_{\ell} b_{-\ell} \times \\
\times & \int_{\mathbb{R}} \int_{\mathbb{R}} e^{\left(\nu+1-\lambda_{1}-\lambda_{2}\right)(t+\tilde{t})} e\left(-\ell e^{2(t+\tilde{t})}\right) \psi_{A}\left(a_{\tilde{t}}\right) d \tilde{t} d t .
\end{aligned}
$$

To simplify this expression further, we set $x=e^{2 t}, y=e^{2 \tilde{t}}$, and

$$
\psi_{A}\left(a_{\tilde{t}}\right)=\psi_{\mathbb{R}}(y) \quad\left(y=e^{2 \tilde{t}}\right) .
$$

Then $d x=2 e^{2 t} d t, d y=2 e^{2 \tilde{t}} d \tilde{t}$, and the normalization 4.24 becomes

$$
\int_{0}^{\infty} \psi_{\mathbb{R}}(y) \frac{d y}{y}=2 .
$$

Putting all the pieces together, we find

$$
\begin{aligned}
& P_{\nu}^{\Gamma}\left(\tau_{1}, \tau_{2}, E_{\nu}\right)= \\
& =\frac{\zeta(\nu+1)}{4} \sum_{\ell \neq 0} a_{\ell} b_{-\ell} \int_{0}^{\infty} \int_{0}^{\infty}(x y)^{\frac{\nu+1-\lambda_{1}-\lambda_{2}}{2}} e(-\ell x y) \psi_{\mathbb{R}}(y) \frac{d y}{y} \frac{d x}{x} .
\end{aligned}
$$

We know from the derivation of this formula that the integral and the sum must converge for $\operatorname{Re} \nu \gg 0$, and indeed they do. Since $\psi_{\mathbb{R}}$ has compact support in $(0, \infty)$, the inner integral is the Fourier transform of a compactly supported function on $\mathbb{R}$. The resulting function of $x$ is smooth at the origin and decays rapidly at infinity. That makes the outer integral converge, provided Re $\nu$ is large enough. A change of variables then shows that the double integral has order of growth $O\left(|\ell|^{\operatorname{Re}\left(\lambda_{1}+\lambda_{2}-\nu-1\right) / 2}\right)$, so the sum does converge, again for $\operatorname{Re} \nu \gg 0$.

If we regard $e(-\ell x y), \ell \neq 0$, not as a function, but as a distribution that vanishes to infinite order at infinity, the inner integral converges for $\operatorname{Re} \nu \gg 0$, and the smoothing process becomes unnecessary. Taking this approach, we make the change of variables $x \mapsto x / y$, which splits off the integral 4.33). Hence

$$
\begin{aligned}
& P_{\nu}^{\Gamma}\left(\tau_{1}, \tau_{2}, E_{\nu}\right)= \\
& =\frac{\zeta(\nu+1)}{2} \sum_{\ell \neq 0} a_{\ell} b_{-\ell} \int_{0}^{\infty} x^{\frac{\nu+1-\lambda_{1}-\lambda_{2}}{2}} e(-\ell x) \frac{d x}{x} \\
& =\frac{\zeta(\nu+1)}{2} \sum_{\ell \neq 0} a_{\ell} b_{-\ell}|\ell|^{\frac{\lambda_{1}+\lambda_{2}-\nu-1}{2}} \int_{0}^{\infty} x^{\frac{\nu+1-\lambda_{1}-\lambda_{2}}{2}} e(-(\operatorname{sgn} \ell) x) \frac{d x}{x} \\
& =\frac{\zeta(2 s)}{2} \sum_{\ell \neq 0} a_{\ell} b_{-\ell}|\ell|^{\frac{\lambda_{1}+\lambda_{2}}{2}-s} \int_{0}^{\infty} x^{s-\frac{\lambda_{1}+\lambda_{2}}{2}} e(-(\operatorname{sgn} \ell) x) \frac{d x}{x} .
\end{aligned}
$$

At the last step, we have expressed $\nu$ in terms of $s$, as in 4.12. 
By definition, the Rankin-Selberg $L$-function of the pair of automorphic distributions $\tau_{1}, \tau_{2}$ is

$$
L\left(s, \tau_{1} \otimes \tau_{2}\right)=\zeta(2 s) \sum_{n>0} a_{n} b_{n} n^{\frac{\lambda_{1}+\lambda_{2}}{2}-s} .
$$

Recall that the Fourier coefficients $a_{n}, b_{n}$ depend on the choice of the embedding parameter $\lambda_{j}$ over $-\lambda_{j}$. The standard $L$-function 2.22 , and $1.2 p$ in the case of modular forms, with $\lambda=1-k$, are defined in terms of the renormalized coefficients $a_{n}|n|^{\lambda / 2}$. For the same reason the renormalized coefficients appear in the Rankin-Selberg $L$-function. To make the connection between 4.35 and the $L$-function, notice that translation by the matrix

$$
r=\left(\begin{array}{cc}
-1 & 0 \\
0 & 1
\end{array}\right)
$$

transforms $\tau_{j} \in\left(V_{\lambda_{j}, \delta_{j}}^{-\infty}\right)^{\Gamma}$, realized as $\tau_{j}(x)$ in terms of the unbounded model, to $(-1)^{\delta_{j}} \tau_{j}(-x)$. Since $r \in \Gamma$, that means $\tau_{j}(-x)=(-1)^{\delta_{j}} \tau_{j}(x)$, i.e.,

$$
a_{-n}=(-1)^{\delta_{1}} a_{n}, \quad b_{-n}=(-1)^{\delta_{2}} b_{n} .
$$

Hence

$$
\begin{aligned}
& \zeta(2 s) \sum_{\ell>0} a_{\ell} b_{-\ell}|\ell|^{\frac{\lambda_{1}+\lambda_{2}}{2}-s}=(-1)^{\delta_{2}} L\left(s, \tau_{1} \otimes \tau_{2}\right), \\
& \zeta(2 s) \sum_{\ell<0} a_{\ell} b_{-\ell}|\ell|^{\frac{\lambda_{1}+\lambda_{2}}{2}-s}=(-1)^{\delta_{1}} L\left(s, \tau_{1} \otimes \tau_{2}\right) .
\end{aligned}
$$

This allows us to re-write 4.35 as

$$
\begin{gathered}
2 P_{\nu}^{\Gamma}\left(\tau_{1}, \tau_{2}, E_{\nu}\right)=L\left(s, \tau_{1} \otimes \tau_{2}\right) \times \\
\times\left\{(-1)^{\delta_{2}} \int_{-\infty}^{0}|x|^{s-\frac{\lambda_{1}+\lambda_{2}}{2}} e(x) \frac{d x}{x}+(-1)^{\delta_{1}} \int_{0}^{\infty}|x|^{s-\frac{\lambda_{1}+\lambda_{2}}{2}} e(x) \frac{d x}{x}\right\} \\
=(-1)^{\delta_{1}} G_{\delta_{1}+\delta_{2}}\left(s-\frac{\lambda_{1}+\lambda_{2}}{2}\right) L\left(s, \tau_{1} \otimes \tau_{2}\right)
\end{gathered}
$$

recall (2.25), and also the relationship $\nu=2 s-1$ between $\nu$ and $s$.

To complete the proof of the functional equation, we combine 4.40 with 4.17) and appeal to the standard Gamma identity $G_{\delta}(s) G_{\delta}(1-s)=(-1)^{\delta}$ :

4.41 Proposition. The Rankin-Selberg L-function satisfies the functional equation

$$
L\left(1-s, \tau_{1} \otimes \tau_{2}\right)=\prod_{\varepsilon_{1}, \varepsilon_{2}= \pm 1} G_{\delta_{1}+\delta_{2}}\left(s+\varepsilon_{1} \frac{\lambda_{1}}{2}+\varepsilon_{2} \frac{\lambda_{2}}{2}\right) L\left(s, \tau_{1} \otimes \tau_{2}\right) .
$$

We have shown that 4.40 has a holomorphic continuation to $\mathbb{C}-\{1\}$, with at most a simple pole at $s=1$. Traditionally one states the functional equation and analytic continuation not for the expression in 4.40 , but rather for Langlands' completed $L$-function 


$$
\Lambda\left(s, \tau_{1} \otimes \tau_{2}\right)=L_{\infty}\left(s, \tau_{1} \otimes \tau_{2}\right) L\left(s, \tau_{1} \otimes \tau_{2}\right),
$$

whose "component at infinity" is a product of Gamma factors that depend on the type of the $\tau_{j}$. If both $\tau_{1}$ and $\tau_{2}$ correspond to Maass forms, then

$$
\text { Maass case: } \begin{aligned}
L_{\infty}\left(s, \tau_{1} \otimes \tau_{2}\right) & =\prod_{\varepsilon_{1}, \varepsilon_{2}= \pm 1} \Gamma_{\mathbb{R}}\left(s+\varepsilon_{1} \frac{\lambda_{1}}{2}+\varepsilon_{2} \frac{\lambda_{2}}{2}+\eta\right), \\
& \text { with } \eta \in\{0,1\}, \quad \eta \equiv \delta_{1}+\delta_{2}(\bmod 2) .
\end{aligned}
$$

Here $\Gamma_{\mathbb{R}}$ denotes the Artin $\Gamma$-factor $\pi^{-s / 2} \Gamma(s / 2)$. If one of the $\tau_{j}$, say $\tau_{2}$ for definiteness, corresponds to a holomorphic cusp form of weight $k$, then

$$
\text { mixed case: } \quad L_{\infty}\left(s, \tau_{1} \otimes \tau_{2}\right)=\Gamma_{\mathbb{C}}\left(s+\frac{\lambda_{1}}{2}+\frac{k-1}{2}\right) \Gamma_{\mathbb{C}}\left(s-\frac{\lambda_{1}}{2}+\frac{k-1}{2}\right),
$$

where $\Gamma_{\mathbb{C}}(s)=2(2 \pi)^{-s} \Gamma(s)$. Finally, when both $\tau_{1}$ and $\tau_{2}$ correspond to holomorphic cusp forms, of weights $k_{1}$ and $k_{2}$, respectively,

$$
\text { modular forms case: } L_{\infty}\left(s, \tau_{1} \otimes \tau_{2}\right)=\Gamma_{\mathbb{C}}\left(s+\frac{k_{1}+k_{2}}{2}-1\right) \Gamma_{\mathbb{C}}\left(s+\frac{\left|k_{1}-k_{2}\right|}{2}\right) \text {. }
$$

In all cases, the functional equation of the previous proposition directly implies the equality of $\Lambda\left(s, \tau_{1} \otimes \tau_{2}\right)$ and $\Lambda\left(1-s, \tau_{1} \otimes \tau_{2}\right)$, up to a sign; this follows from standard Gamma identities, in particular the identity $G_{\delta}(s) G_{\delta}(1-s)=(-1)^{\delta}$ and the Legendre duplication formula.

Just as important as the functional equation is the assertion of holomorphy: both $L\left(s, \tau_{1} \otimes \tau_{2}\right)$ and $\Lambda\left(s, \tau_{1} \otimes \tau_{2}\right)$ are holomorphic except for potential first order poles at $s=0$ and $s=1$. For the uncompleted $L$-function this follows from a classical argument of Jacquet [6, Lemma 14.7.5]. His argument does not require any detailed calculations, and holds in great generality.

Once $L\left(s, \tau_{1} \otimes \tau_{2}\right)$ is known to be holomorphic on $\mathbb{C}-\{0,1\}$, one can deduce the holomorphy of $\Lambda\left(s, \tau_{1} \otimes \tau_{2}\right)$ on $\mathbb{C}-\{0,1\}$ from the results of this section, as follows. Because of the functional equation, it suffices to rule out poles in the region $\{\operatorname{Re} s \geq 1 / 2, s \neq 1\}$. In effect, we must show that all poles of $L_{\infty}\left(s, \tau_{1} \otimes \tau_{2}\right)$ with $\operatorname{Re} s \geq 1 / 2$ are compensated by zeroes of $L\left(s, \tau_{1} \otimes \tau_{2}\right)$. This is an issue only in the Maass case: modular forms have weights at least 2 , and the parameter $\lambda$ of a Maass form necessarily lies in the region $\{|\operatorname{Re} \lambda|<1 / 2\}$. In the Maass case, only one of the four Gamma factors in 4.43 can have a pole with $\operatorname{Re} s \geq 1 / 2$. Maass forms correspond to irreducible principal series representations, which involve $\lambda_{j}$ and $-\lambda_{j}$ symmetrically. We can therefore assume that $\operatorname{Re} \lambda_{j} \geq 0$, in which case the pole can only come from the factor $\Gamma_{\mathbb{R}}\left(s-\frac{\lambda_{1}+\lambda_{2}}{2}\right)$, with $\eta=0$, and must occur at $s=\frac{\lambda_{1}+\lambda_{2}}{2}$. But then $\delta_{1}=\delta_{2}$, and $G_{\delta_{1}+\delta_{2}}\left(s-\frac{\lambda_{1}+\lambda_{2}}{2}\right)=G_{0}\left(s-\frac{\lambda_{1}+\lambda_{2}}{2}\right)$ also has a pole at $s=\frac{\lambda_{1}+\lambda_{2}}{2}$. We know that (4.40) is holomorphic on $\mathbb{C}-\{0,1\}$, thus forcing $L\left(s, \tau_{1} \otimes \tau_{2}\right)$ to vanish at $s=\frac{\lambda_{1}+\lambda_{2}}{2}$, as was to be shown.

\section{Exterior Square on $G L(4)$}

Recall that if $F$ is a Hecke eigenform on $G L(n, \mathbb{Z}) \backslash G L(n, \mathbb{R})$, or more generally, on the quotient of $G L(n, \mathbb{R})$ by a congruence subgroup, the standard 
$L$-function of $F$ has an Euler product

$$
L(s, F)=\prod_{p} \prod_{j=1}^{n}\left(1-\alpha_{p, j} p^{-s}\right)^{-1} .
$$

The exterior square $L$-function is then defined as an Euler product

$$
L\left(s, F, E x t^{2}\right)=\prod_{p} L_{p}\left(s, F, E x t^{2}\right),
$$

whose factor at any unramified prime $p$ equals

$$
L_{p}\left(s, F, E x t^{2}\right)=\prod_{1 \leq j<k \leq n}\left(1-\alpha_{p, j} \alpha_{p, k} p^{-s}\right)^{-1} .
$$

The appropriate definition of the factors $L_{p}\left(s, F, E x t^{2}\right)$ corresponding to the finitely many ramified primes is still a subtle issue. Harris and Taylor recently exhibited local factors for the ramified primes that are consistent with Langlands functoriality principles, in their proof of the local Langlands conjectures for $G L(n)$. However, Shahidi had much earlier given a separate definition, which by all expectations agrees with the one provided by Harris-Taylor, though the agreement of the two definitions is not obvious. Shahidi furthermore proved that the $L$-function with his definition of the ramified factors satisfies a functional equation of the type Langlands predicted. Since there can only be one definition which obeys this functional equation, the potential discrepancy between the Harris-Taylor and Shahidi definitions poses no problem from the point of view of $L$-functions, though it still is a problem for the group-theoretic definition of the Langlands conjectures. In any case, an argument which produces the analytic continuation and functional equation of $L\left(s, F, E x t^{2}\right)$ must give a definition which agrees with Shahidi's.

In our paper [15], we carry out the archimedean analysis of the exterior square $L$-function for $G L(n)$; we establish the holomorphy of the partial $L$ function $L_{S}(s, F)$ and its completion at infinity $\Lambda_{S}(s, F)$, in both cases with the factors in (5.1) corresponding to the set $S$ of ramified primes omitted. To keep the discussion simple, we avoid the problem of ramification in the present paper by treating only the full level subgroup $G L(4, \mathbb{Z}) \subset G L(4, \mathbb{R})$.

By necessity, the notation in this section will not completely agree with that of the earlier sections; in particular, we now set

$$
G=G L(4, \mathbb{R}), G_{0}=S L^{ \pm}(2, \mathbb{R}), \quad \Gamma=G L(4, \mathbb{Z}), \quad \Gamma_{0}=S L^{ \pm}(2, \mathbb{Z}) .
$$

We shall also work with the subgroups

$$
\begin{gathered}
G_{1}=\left\{\left(\begin{array}{cc}
g_{1} & 0 \\
0 & g_{2}
\end{array}\right) \mid g_{1}, g_{2} \in G L(2, \mathbb{R})\right\} \subset G, \\
\Gamma_{1}=\left\{\left(\begin{array}{ll}
\gamma & 0 \\
0 & \gamma
\end{array}\right) \mid \gamma \in G L(2, \mathbb{Z})\right\} \subset \Gamma, \\
U=\left\{\left(\begin{array}{ll}
1 & u \\
0 & 1
\end{array}\right) \mid u \in M_{2 \times 2}(\mathbb{R})\right\} \subset G .
\end{gathered}
$$


Note that $G_{1} \simeq G L(2, \mathbb{R}) \times G L(2, \mathbb{R})$ contains $\Gamma_{1} \simeq G L(2, \mathbb{Z})$, but not as an arithmetic subgroup.

Again we let $B \subset G$ denote the lower triangular Borel subgroup, and we define $B_{1}=G_{1} \cap B$. Each pair

$$
(\mu, \eta) \in \mathbb{C}^{4} \times(\mathbb{Z} / 2 \mathbb{Z})^{4}
$$

determines a character $\chi_{\mu, \eta}: B \rightarrow \mathbb{C}^{*}$,

$$
\chi_{\mu, \eta}\left(a_{i, j}\right)=\prod_{1 \leq i \leq 4}\left|a_{i, i}\right|^{\mu_{i}}\left(\operatorname{sgn} a_{i, i}\right)^{\eta_{i}},
$$

and by restriction also a character $\chi_{\mu, \eta}: B_{1} \rightarrow \mathbb{C}^{*}$. For $G=G L(4, \mathbb{R})$,

$$
\rho=\left(\frac{3}{2}, \frac{1}{2},-\frac{1}{2},-\frac{3}{2}\right)
$$

represents the half sum of the positive roots. In analogy to 3.12 ,

$$
W_{\mu, \eta}^{-\infty}=\left\{\tau \in C^{-\infty}(G) \mid \tau(g b)=\chi_{\mu-\rho, \eta}\left(b^{-1}\right) \tau(g) \text { for all } g \in G, b \in B\right\}
$$

is the space of distribution vectors for a generic principal series representation of $G$. Principal series representations of $G_{1} \simeq G L(2, \mathbb{R}) \times G L(2, \mathbb{R})$ are induced from $B_{1}$, and hence also parameterized by pairs $(\mu, \eta) \in \mathbb{C}^{4} \times(\mathbb{Z} / 2 \mathbb{Z})^{4}$,

$$
V_{\mu, \eta}^{-\infty}=\left\{\tau \in C^{-\infty}\left(G_{1}\right) \mid \tau(g b)=\chi_{\mu-\rho, \eta}\left(b^{-1}\right) \tau(g) \text { for all } g \in G_{1}, b \in B_{1}\right\} .
$$

Our current use of the notation $V_{\mu, \eta}^{-\infty}$ is not consistent with $(3.12$. Not only is $G_{1}$ a product of two copies of $G L(2, \mathbb{R})$, but the representations we consider need not be trivial on the center of $G L(2, \mathbb{R})$, in contrast to the situation in section 3, where we considered only automorphic distributions for $P G L(2, \mathbb{R})$. However, the $\rho$-shift in (5.10) is consistent with (3.12): the quantity $\rho$ defined in (5.8) restricts to the corresponding quantities for the two factors of $G_{1} \simeq$ $G L(2, \mathbb{R}) \times G L(2, \mathbb{R})$.

The arithmetic group $\Gamma$ intersects $U \simeq \mathbb{R}^{4}$ in a lattice, so $(\Gamma \cap U) \backslash U$ is compact. That makes it possible to define the operator

$$
\begin{aligned}
A:\left(W_{\mu, \eta}^{-\infty}\right)^{\Gamma} & \longrightarrow\left(V_{\mu, \eta}^{-\infty}\right)^{\Gamma_{1}}, \\
A \tau(g) & =\int_{(\Gamma \cap U) \backslash U} \tau(u g) e(-\operatorname{tr} u) d u \quad\left(g \in G_{1}\right) .
\end{aligned}
$$

What matters is the fact that the $U \cdot G_{1}$-orbit of the identity coset in $G / B$ is open. One can therefore restrict any $\tau \in\left(W_{\mu, \eta}^{-\infty}\right)^{\Gamma}$ to this open subset, and then further to $G_{1}$, once the dependence on the variable $u \in U$ has been smoothed out by taking a single Fourier component. The restriction to $G_{1}$ still transforms according to $\chi_{\mu, \eta}^{-1}$ under right translation by elements of $B_{1}=G_{1} \cap B$. This makes $A \tau$ lie in $V_{\mu, \eta}^{-\infty}$. Conjugation by any $\gamma \in \Gamma_{1}$ preserves the character $u \mapsto e(-\operatorname{tr} u)$ of $U$ and the lattice $\Gamma \cap U$. Since $\Gamma_{1} \subset \Gamma$, the $\Gamma$-invariance of $\tau$ ensures the $\Gamma_{1}$-invariance of $A \tau$. 
We now consider a particular cuspidal $\tau \in\left(W_{\mu, \eta}^{-\infty}\right)^{\Gamma}$. Since $\Gamma$ contains the center of $S L^{ \pm}(4, \mathbb{R})$, any such $\tau$ must vanish identically unless

$$
\sum_{1 \leq j \leq 4} \eta_{j}=0 \text { in } \mathbb{Z} / 2 \mathbb{Z}
$$

We shall also suppose that

$$
\sum_{1 \leq j \leq 4} \mu_{j}=0
$$

This is not a serious restriction: it holds automatically when $\tau$ arises from a discrete summand of $L^{2}\left(\Gamma \backslash G / Z_{G}^{0}\right)$, as in (3.1). Even when that is not the case, we can modify $\tau$ by twisting with an appropriate character of $Z_{G}^{0}$, without destroying the $\Gamma$-invariance.

In section 3, we described the pairing of three $P G L(2, \mathbb{Z})$-automorphic distributions on $P G L(2, \mathbb{R})$. By limiting ourselves to the case of $P G L(2, \mathbb{R})$ we avoided some notational complications in (3.21) and (3.24), without essential loss of generality: in the case of full level, $-1 \in G L(2, \mathbb{Z})$ must act trivially on any automorphic distribution. In the current setting, we do need the pairing for triples of automorphic distributions on $G L(2, \mathbb{R})$. Theorem 3.33 remains correct as stated in this more general situation, provided the integration is performed over $S L^{ \pm}(2, \mathbb{Z}) \backslash S L^{ \pm}(2, \mathbb{R})$ - the center of $G L(2, \mathbb{R})$ is noncompact and remains noncompact even modulo $G L(2, \mathbb{Z})$. The statement requires the $\Gamma$-invariance of all three of the arguments $\tau_{j}$ of the pairing $P$. Formally, at least, invariance under the diagonal action of $\Gamma$ on the three arguments suffices to produce a $\Gamma$-invariant integrand for the outer integral in theorem 3.33. It is the proof of rapid decay that forces us to assume $\Gamma$-invariance of each factor. In the present setting, $A \tau$ arises from a cuspidal automorphic distribution $\tau$ on $G L(4, \mathbb{R})$. It is not difficult to adapt the proof of theorem 3.33 to this case: after smoothing by some $\psi \in C_{c}^{\infty}\left(G_{0}\right)$, the product of $A \tau$ with the Eisenstein series $E_{\nu}$ does decay rapidly along the cusp.

We again define the Eisenstein series $E_{\nu}$ by the formula 4.2 , but now summing over $\Gamma_{0} /\left(\Gamma_{0}\right)_{\infty}$; since $\left.-1 \in\left(\Gamma_{0}\right)_{\infty}, 4.54 .7\right)$ remain correct. We should remark that the pairing of three automorphic distributions on $G L(2, \mathbb{R})$ vanishes identically unless $-1 \in G L(2, \mathbb{R})$ acts trivially under the diagonal action. The parity condition (5.12) implies that -1 acts trivially under the diagonal action on $A \tau$. But -1 also acts trivially on delta function $\delta_{\infty}$, and hence on the Eisenstein series $E_{\nu}$. In short, the parity condition imposed by the action of the center is satisfied in our situation. We have assembled all ingredients to make sense of

$$
P_{\nu}^{\Gamma_{0}}\left(A \tau, E_{\nu}\right)=\int_{\Gamma_{0} \backslash G_{0}} \int_{G_{0}} A \tau\left(\begin{array}{cc}
g h f_{1} & 0 \\
0 & g h f_{2}
\end{array}\right) E_{\nu}\left(g h f_{3}\right) \psi(h) d h d g .
$$

As a function of $\nu$ this is holomorphic, except for a potential first order pole at $\nu=1$. What we said in section 3 about the intertwining operator $J_{\nu}$ and its interaction with the pairing remains valid, except for the parity subscripts of 
the Gamma factors in 4.15) and 4.17), since we now work on $G L(2, \mathbb{R})$. The roles of $\lambda_{1}$ and $\lambda_{2}$ are played by, respectively, $\mu_{1}-\mu_{2}$ and $\mu_{3}-\mu_{4}$, as can be seen by comparing the definition 5.10 of $V_{\mu, \eta}^{-\infty}$ to the definition 3.12 . Thus, and because of $5.13, \frac{\lambda_{1}-\lambda_{2}}{2}$ corresponds to $\mu_{1}+\mu_{4}$ and $\frac{\lambda_{2}-\lambda_{1}}{2}$ corresponds to $\mu_{2}+\mu_{3}$. This explains the arguments of the Gamma factors in the identity

$$
\begin{aligned}
& P_{\nu}^{\Gamma_{0}}\left(A \tau, E_{\nu}\right)=(-1)^{\eta_{2}+\eta_{3}} \times \\
& \quad \times G_{\eta_{1}+\eta_{4}}\left(\mu_{1}+\mu_{4}-\frac{\nu-1}{2}\right) G_{\eta_{2}+\eta_{3}}\left(\mu_{2}+\mu_{3}-\frac{\nu-1}{2}\right) P_{-\nu}^{\Gamma_{0}}\left(A \tau, E_{-\nu}\right),
\end{aligned}
$$

which takes the place of 4.17 in the current setting. In the special case when $\eta_{1}=\eta_{2}$ and $\eta_{3}=\eta_{4}$ - i.e, when the action of $G_{1} \cong G L(2, \mathbb{R}) \times G L(2, \mathbb{R})$ on $A \tau$ drops to $P G L(2, \mathbb{R}) \times P G L(2, \mathbb{R})-(5.15$ agrees with in 4.17 , as it must. In the remaining cases the identity is deduced from the appropriate variant of 4.16; for details see 15 .

The identity 5.15 is the source of the functional equation of the exterior square $L$-function, just as 4.17) was the source of the functional equation for the Rankin-Selberg $L$-function $L\left(s, \tau_{1} \otimes \tau_{2}\right)$. To make the connection between the identity 5.15) and the exterior square $L$-function, we need to consider the Fourier expansion of $\tau$ on

$$
N=\left\{n(x, u, v)=\left(\begin{array}{cccc}
1 & x_{1} & u_{1} & v \\
0 & 1 & x_{2} & u_{2} \\
0 & 0 & 1 & x_{3} \\
0 & 0 & 0 & 1
\end{array}\right) \mid x \in \mathbb{R}^{3}, u \in \mathbb{R}^{2}, v \in \mathbb{R}\right\} .
$$

Since the $N$-orbit through the identity coset in $G / B$ is open, it is legitimate to restrict $\tau$ to $N$. This restriction is $(\Gamma \cap N)$-invariant, which allows us to regard $\tau$ as lying in $C^{-\infty}((\Gamma \cap N) \backslash N)$. Every $(\Gamma \cap N)$-invariant smooth function on $G$, and dually every $(\Gamma \cap N)$-invariant distribution, has a Fourier expansion with components indexed by - roughly speaking - the irreducible unitary representations of $N$. For the one dimensional, or abelian, representations this is literally true, but typically infinite dimensional representation contribute more than once, but finitely often. The non-abelian Fourier components will turn out not to matter for our purposes. Thus we write

$$
\tau(n(x, u, v))=\sum_{1 \leq j \leq 3} a_{n_{1}, n_{2}, n_{3}} e\left(n_{j} x_{j}\right)+\ldots,
$$

with ... denoting the sum of the non-abelian Fourier components of $\tau$. The $a_{n_{1}, n_{2}, n_{3}}$ with positive indices $n_{j}$ determine all the others:

$$
a_{\epsilon_{1} n_{1}, \epsilon_{2} n_{2}, \epsilon_{3} n_{3}}=\epsilon_{1}^{\eta_{1}} \epsilon_{2}^{\eta_{1}+\eta_{2}} \epsilon_{3}^{\eta_{1}+\eta_{2}+\eta_{3}} a_{n_{1}, n_{2}, n_{3}} \quad\left(\epsilon_{j} \in\{ \pm 1\}\right) .
$$

Indeed, $\tau$ is invariant under the action of all diagonal matrices with entries \pm 1 , since $\Gamma$ contains these. Each of them acts on $N$ by conjugation, which has the effect of reversing the signs of some of the coordinates. One can then use (5.7) to determine how the $a_{n_{1}, n_{2}, n_{3}}$ change when the signs of one or more of the indices is flipped. 
When $\tau$ is a Hecke eigendistribution, the Fourier coefficients $a_{n_{1}, n_{2}, n_{3}}$ are related to the Hecke eigenvalues. Specifically, $k^{\mu_{1}+\mu_{2}} a_{1, k, 1}$ is the eigenvalue of the Hecke operator $T_{1, k, 1}$. The eigenvalues for Hecke operators indexed by unramified primes can be expressed in terms of the $\alpha_{j, p}$ in (5.1) 20]. Jacquet and Shalika [7, §2] have used this expression to identify the factors $L_{p}\left(s, \tau, E x t^{2}\right)$ for unramified primes $p$ in terms of the Hecke eigenvalues - in complete generality for all $n$, not just $n=4$. In the case of $G L(4)$,

$$
L_{p}\left(s, \tau, E x t^{2}\right)=\left(1-p^{-2 s}\right)^{-1} \sum_{k \geq 0} a_{1, p^{k}, 1} p^{k\left(\mu_{1}+\mu_{2}-s\right)} .
$$

At full level, when there are no ramified primes, the Euler product of the local factors for all primes, as in (5.2), expresses the exterior square $L$-function as

$$
L\left(s, \tau, E x t^{2}\right)=\zeta(2 s) \sum_{n \geq 1} a_{1, n, 1} n^{\mu_{1}+\mu_{2}-s} .
$$

One can use this as the definition of the exterior square $L$-function whether or not $\tau$ is a Hecke eigendistribution.

5.21 Lemma. When $s$ and $\nu$ are related by the equation $2 s=\nu+1$,

$$
P_{\nu}^{\Gamma_{0}}\left(A \tau, E_{\nu}\right)=2(-1)^{\eta_{2}} G_{\eta_{1}+\eta_{2}}\left(s-\mu_{1}-\mu_{2}\right) G_{\eta_{1}+\eta_{3}}\left(s-\mu_{1}-\mu_{3}\right) L\left(s, \tau, E x t^{2}\right) .
$$

Since the proof is lengthy, we shall first deduce the functional equation, which follows from the lemma in combination with (5.15), (5.12 5.13$)$, and the standard Gamma identity $G_{\delta}(s) G_{\delta}(1-s)=(-1)^{\delta}$ :

$$
\text { 5.22 Proposition. } L\left(1-s, \tau, E x t^{2}\right)=\prod_{1 \leq i<j \leq 4} G_{\eta_{i}+\eta_{j}}\left(s-\mu_{i}-\mu_{j}\right) L\left(s, \tau, E x t^{2}\right) \text {. }
$$

This result is originally due to Kim $[9]$ and, in the special case when $W_{\mu, \eta}$ belongs to the spherical principal series, to Stade 21. We refer the reader to our paper 15 for a discussion of the history of the exterior square $L$-function for $G L(n)$.

The usual statement of functional equation relates the exterior square $L$ function $L\left(s, \tau, E x t^{2}\right)$ for $G L(n)$ to that of the dual automorphic distribution $\widetilde{\tau}$. In our case, with $G=G L(4, \mathbb{R})$, these two $L$-functions coincide; that makes it possible to state the functional equation without reference to $\widetilde{\tau}$.

Just as in the case of the Rankin-Selberg $L$-function for $G L(2)$, Jacquet's general argument implies that $L\left(s, \tau, E x t^{2}\right)$ is holomorphic, except for possible first order poles at $s=0$ and $s=1$ 15. The fact that $P_{\nu}^{\Gamma_{0}}\left(A \tau, E_{\nu}\right)$ is holomorphic, together with an analysis of the poles and zeros of the Gamma factors, establishes the holomorphy of the completed exterior square $L$-function, again with the possible exception of first order poles at 0 and 1 . We conclude our paper with the proof of the lemma.

Proof of Lemma 5.21. Recall the notational conventions (5.4); in particular $G_{0}^{0}=S L(2, \mathbb{R})$ denotes the identity component of $G_{0}=S L^{ \pm}(2, \mathbb{R})$. We shall 
suppose that the smoothing function $\psi$ is supported on $G_{0}^{0}$, as we did in section 4 . We also impose the $K$-invariance condition (4.23) and define $\psi_{A}$ as we did in (4.24). In section 3 we had pointed out that the expression (3.34) is smooth as function of all three variables. For the same reason

$$
\left(g_{1}, g_{2}, g_{3}\right) \mapsto \int_{G_{0}^{0}} A \tau\left(\begin{array}{cc}
g_{1} h f_{1} & 0 \\
0 & g_{2} h f_{2}
\end{array}\right) \delta_{\infty}\left(g_{3} h f_{3}\right) \psi(h) d h
$$

is a $C^{\infty}$ function on $G_{0} \times G_{0} \times G_{0}$. It is also an eigenfunction of the Casimir operator in each of the three variables, of moderate growth since $\tau$ and $\delta_{\infty}$ are distribution vectors. The cuspidality of $\tau$ implies that the restriction of this function to the triple diagonal decays rapidly in the cuspidal directions. We can therefore set $g_{1}=g_{2}=g_{3}=g$ and integrate with respect to $g$ over the quotient $\Gamma_{0, \infty} \backslash G_{0}$, with $\Gamma_{0, \infty}=\left\{\gamma \in \Gamma_{0} \mid \gamma \infty=\infty\right\}$.

In analogy with (4.18), we insert the definition (4.2) of $E_{\nu}$ into (5.14) and unfold: for $\operatorname{Re} \nu \gg 0$,

$$
P_{\nu}^{\Gamma_{0}}\left(A \tau, E_{\nu}\right)=\zeta(\nu+1) \int_{\Gamma_{0, \infty} \backslash G_{0}} \int_{G_{0}} A \tau\left(\begin{array}{cc}
g h f_{1} & 0 \\
0 & g h f_{2}
\end{array}\right) \delta_{\infty}\left(g h f_{3}\right) \psi(h) d h d g,
$$

The justification of this step hinges on two facts. First of all, the function (5.23) has moderate growth, as was just pointed out Secondly, we know the behavior of $\delta_{\infty}$ under left translation by elements of $A$. From here on we can justify the unfolding exactly as in section 4 . In (5.24) we can replace $G_{0}$ in the inner integral by the identity component $G_{0}^{0}$ on which $\psi$ is supported. Since $\Gamma_{0, \infty}$ meets both connected components of $G_{0}$, we can also replace $G_{0}$ by $G_{0}^{0}$ in the outer integral, provided we simultaneously replace $\Gamma_{0, \infty}$ by $\Gamma_{0, \infty}^{0}=\Gamma_{0, \infty} \cap G_{0}^{0}$. We parameterize $G_{0}^{0}$ by the Iwasawa decomposition $g=n_{x} a k$ - recall (3.17) and 4.20 4.21) . To avoid confusion, we now let $N_{0}, A_{0}, K_{0}$ denote the subgroups of $G_{0}^{0}=S L(2, \mathbb{R})$ analogous to $N, A, K$ in sections 3 and 4 . Note that $\Gamma \cap N_{0}$ has index 2 in $\Gamma_{0, \infty}^{0}$, which also contains -1 , so $\left(\Gamma \cap N_{0}\right) \backslash N_{0} A_{0} K_{0}$ covers $\Gamma_{0, \infty}^{0} \backslash G_{0}^{0}$ twice. Thus

$$
\begin{aligned}
& P_{\nu}^{\Gamma_{0}}\left(A \tau, E_{\nu}\right)=2 \zeta(\nu+1) \times \\
& \quad \times \int_{A_{0}} \int_{0}^{1} \int_{G_{0}^{0}} e^{-2 \rho}(a) A \tau\left(\begin{array}{cc}
n_{x} a h f_{1} & 0 \\
0 & n_{x} a h f_{2}
\end{array}\right) \delta_{\infty}\left(n_{x} a h f_{3}\right) \psi(h) d h d x d a ;
\end{aligned}
$$

we have legitimately omitted the integration over the Iwasawa component $k$ because $\psi$ is $K$-invariant.

Recall the definition 5.11) of $A \tau$. It will be convenient to replace $\tau$ by $\tau^{0}$, defined by the formula

$$
\tau^{0}(g)=\int_{\left(\Gamma \cap Z_{N}\right) \backslash Z_{N}} \tau(n g) d n=\int_{0}^{1} \tau\left(\left(\begin{array}{llll}
1 & 0 & 0 & v \\
0 & 1 & 0 & 0 \\
0 & 0 & 0 \\
0 & 0 & 0 & 1
\end{array}\right) g\right) d v,
$$

with $Z_{N}=$ center of $N$. Then $\tau^{0}$ is invariant under left translation by elements of $Z_{N}$, and by elements of $\Gamma \cap N$. We shall also need to know that 


$$
\tau^{0}\left(s_{2,3} g\right)=\tau^{0}(g) \quad \text { for all } g \in G, \quad \text { with } s_{2,3}=\left(\begin{array}{llll}
1 & 0 & 0 & 0 \\
0 & 0 & 1 & 0 \\
0 & 1 & 0 & 0 \\
0 & 0 & 0 & 1
\end{array}\right) \text {. }
$$

Indeed, $s_{2,3}$ is contained in $\Gamma$ and commutes with the one parameter group over which $\tau$ is averaged to produce $\tau^{0}$. The passage from $\tau^{0}$ to $A \tau$ involves averaging over three more variables,

$$
A \tau(g)=\int_{\mathbb{R}^{3} / \mathbb{Z}^{3}} \tau^{0}\left(\left(\begin{array}{cccc}
1 & 0 & u_{1} & 0 \\
0 & 1 & x_{2} & u_{2} \\
0 & 0 & 1 & 0 \\
0 & 0 & 0 & 1
\end{array}\right) g\right) e\left(-u_{1}-u_{2}\right) d x_{2} d u_{1} d u_{2} .
$$

Since

$$
\left(\begin{array}{cccc}
1 & 0 & u_{1} & 0 \\
0 & 1 & x_{2} & u_{2} \\
0 & 0 & 1 & 0 \\
0 & 0 & 0 & 1
\end{array}\right)\left(\begin{array}{cccc}
1 & x & 0 & 0 \\
0 & 1 & 0 & 0 \\
0 & 0 & 1 & x \\
0 & 0 & 0 & 1
\end{array}\right)=\left(\begin{array}{cccc}
1 & x & u_{1}-x x_{2} & u_{1} x \\
0 & 1 & 0 & u_{2}+x x_{2} \\
0 & 0 & 1 & x \\
0 & 0 & 0 & 1
\end{array}\right)\left(\begin{array}{cccc}
1 & 0 & 0 & 0 \\
0 & 1 & x_{2} & 0 \\
0 & 0 & 1 & 0 \\
0 & 0 & 0 & 1
\end{array}\right),
$$

the equations 5.255 and $(5.28)$ imply

$$
\begin{aligned}
& P_{\nu}^{\Gamma_{0}}\left(A \tau, E_{\nu}\right)=2 \zeta(\nu+1) \times \\
& \quad \times \int_{A_{0}} \int_{0}^{1} \int_{G_{0}^{0}} \int_{\mathbb{R}^{3} / \mathbb{Z}^{3}} \tau^{0}\left(\left(\begin{array}{cccc}
1 & x & u_{1} & 0 \\
0 & 1 & 0 & u_{2} \\
0 & 0 & 1 & x \\
0 & 0 & 0 & 1
\end{array}\right)\left(\begin{array}{cccc}
1 & 0 & 0 & 0 \\
0 & 1 & x_{2} & 0 \\
0 & 0 & 1 & 0 \\
0 & 0 & 0 & 1
\end{array}\right)\left(\begin{array}{ccc}
a h f_{1} & 0 \\
0 & a h f_{2}
\end{array}\right)\right) \\
& \quad \times e^{-2 \rho}(a) e\left(-u_{1}-u_{2}\right) \delta_{\infty}\left(n_{x} a h f_{3}\right) \psi(h) d x_{2} d u_{1} d u_{2} d h d x d a .
\end{aligned}
$$

We appeal to the invariance of $\tau^{0}$ under the center of $N$ to justify setting the $(1,4)$-entry of the first matrix in the argument of $\tau^{0}$ equal to zero.

The variable of integration $x$ occurs three times in 5.30 . Since $\delta_{\infty}$ is $N_{0}$-invariant, we may as well drop the factor $n_{x}$ in its argument. When we omit the integration with respect to $x$ and treat the remaining instances of $x$ as two separate variables, the integrand - after averaging over $\mathbb{R}^{3} / \mathbb{Z}^{3}$ and smoothing with respect to $\psi-$ is a $C^{\infty}$ function of those two variables; this follows from the fact that 5.23 is separately smooth in all three arguments. We can therefore replace the single integral with respect to $x$ by a double integral, provided we multiply the integrand by the delta function, evaluated on the difference of the two variables. Since

$$
\left(\begin{array}{llll}
1 & k & 0 & 0 \\
0 & 1 & 0 & 0 \\
0 & 0 & 1 & \ell \\
0 & 0 & 0 & 1
\end{array}\right)\left(\begin{array}{cccc}
1 & x_{1} & u_{1} & 0 \\
0 & 1 & 0 & u_{2} \\
0 & 0 & 1 & x_{3} \\
0 & 0 & 0 & 1
\end{array}\right) \equiv\left(\begin{array}{cccc}
1 & x_{1}+k & u_{1} & 0 \\
0 & 1 & 0 & u_{2} \\
0 & 0 & 1 & x_{3}+\ell \\
0 & 0 & 0 & 1
\end{array}\right)
$$

modulo the center of $N$, the integrand in 5.30 is separately periodic when the remaining instances of the variable $x$ are uncoupled. The sum

$$
\delta_{0}\left(x_{1}-x_{3}\right)=\sum_{\ell \in \mathbb{Z}} e\left(\ell\left(x_{1}-x_{3}\right)\right)
$$

represents the "delta function along the diagonal" in $\mathbb{R}^{2} / \mathbb{Z}^{2}$. Thus, in view of what we just said,

$$
\begin{aligned}
P_{\nu}^{\Gamma_{0}}(A \tau, & \left.E_{\nu}\right)=2 \zeta(\nu+1) \sum_{\ell \in \mathbb{Z}} \int_{A_{0}} \int_{\mathbb{R}^{2} / \mathbb{Z}^{2}} \int_{G_{0}^{0}} \int_{\mathbb{R}^{3} / \mathbb{Z}^{3}} e\left(\ell\left(x_{1}-x_{3}\right)\right) \times \\
& \times \tau^{0}\left(\left(\begin{array}{cccc}
1 & x_{1} & u_{1} & 0 \\
0 & 1 & 0 & u_{2} \\
0 & 0 & 1 & x_{3} \\
0 & 0 & 0 & 1
\end{array}\right)\left(\begin{array}{cccc}
1 & 0 & 0 & 0 \\
0 & 1 & x_{2} & 0 \\
0 & 0 & 1 & 0 \\
0 & 0 & 0 & 1
\end{array}\right)\left(\begin{array}{cc}
a h f_{1} & 0 \\
0 & a h f_{2}
\end{array}\right)\right) e^{-2 \rho}(a) \\
& \times e\left(-u_{1}-u_{2}\right) \delta_{\infty}\left(a h f_{3}\right) \psi(h) d x_{2} d u_{1} d u_{2} d h d x_{1} d x_{3} d a
\end{aligned}
$$


We use the matrix identity

$$
\left(\begin{array}{llll}
1 & 0 & 0 & 0 \\
0 & 1 & \ell & 0 \\
0 & 0 & 1 & 0 \\
0 & 0 & 0 & 1
\end{array}\right)\left(\begin{array}{cccc}
1 & x_{1} & u_{1} & 0 \\
0 & 1 & 0 & u_{2} \\
0 & 0 & 1 & x_{3} \\
0 & 0 & 0 & 1
\end{array}\right)\left(\begin{array}{cccc}
1 & 0 & 0 & 0 \\
0 & 1 & x_{2} & 0 \\
0 & 0 & 1 & 0 \\
0 & 0 & 0 & 1
\end{array}\right)=\left(\begin{array}{ccccc}
1 & x_{1} & u_{1}-\ell x_{1} & 0 \\
0 & 1 & 0 & u_{2}+\ell x_{3} \\
0 & 0 & 1 & x_{3} \\
0 & 0 & 0 & 1
\end{array}\right)\left(\begin{array}{cccc}
1 & 0 & 0 & 0 \\
0 & 1 & x_{2}+\ell & 0 \\
0 & 0 & 1 & 0 \\
0 & 0 & 0 & 1
\end{array}\right),
$$

the $(\Gamma \cap N)$-invariance of $\tau^{0}$, and the change of variables $u_{1} \mapsto u_{1}+\ell x_{1}$, $u_{2} \mapsto u_{2}-\ell x_{3}$ to eliminate the factor $e\left(\ell\left(x_{1}-x_{3}\right)\right)$ in 5.33$)$ while simultaneously replacing $x_{2}$ by $x_{2}+\ell$. We then combine the $x_{2}$-integral over $\left\{0 \leq x_{2} \leq 1\right\}$ with the sum over $\ell$ into a single integral over $\mathbb{R}$ :

$$
\begin{aligned}
& P_{\nu}^{\Gamma_{0}}\left(A \tau, E_{\nu}\right)=2 \zeta(\nu+1) \times \\
& \quad \times \int_{A_{0}} \int_{\mathbb{R}^{2} / \mathbb{Z}^{2}} \int_{G_{0}^{0}} \int_{\mathbb{R}} \int_{\mathbb{R}^{2} / \mathbb{Z}^{2}} \tau^{0}\left(\left(\begin{array}{cccc}
1 & x_{1} & u_{1} & 0 \\
0 & 1 & 0 & u_{2} \\
0 & 0 & 1 & x_{3} \\
0 & 0 & 0 & 1
\end{array}\right)\left(\begin{array}{cccc}
1 & 0 & 0 & 0 \\
0 & 1 & y & 0 \\
0 & 0 & 1 & 0 \\
0 & 0 & 0 & 1
\end{array}\right)\left(\begin{array}{cc}
a h f_{1} & 0 \\
0 & a h f_{2}
\end{array}\right)\right) \\
& \quad \times e^{-2 \rho}(a) e\left(-u_{1}-u_{2}\right) \delta_{\infty}\left(a h f_{3}\right) \psi(h) d u_{1} d u_{2} d y d h d x_{1} d x_{3} d a .
\end{aligned}
$$

The symbol $y$ instead of $x_{2}$ is meant to emphasize the new role of this variable.

Recall the invariance of $\tau^{0}$ under $s_{2,3}$, as defined in (5.27). Conjugating $s_{2,3}$ across the first matrix in the argument of $\tau^{0}$ has the effect of switching the roles of the $x_{i}$ and the $u_{j}$,

$$
\begin{aligned}
& P_{\nu}^{\Gamma_{0}}\left(A \tau, E_{\nu}\right)=2 \zeta(\nu+1) \times \\
& \quad \times \int_{A_{0}} \int_{\mathbb{R}^{2} / \mathbb{Z}^{2}} \int_{G_{0}^{0}} \int_{\mathbb{R}} \int_{\mathbb{R}^{2} / \mathbb{Z}^{2}} \tau^{0}\left(\left(\begin{array}{cccc}
1 & x_{1} & u_{1} & 0 \\
0 & 1 & 0 & u_{2} \\
0 & 0 & 1 & x_{3} \\
0 & 0 & 0 & 1
\end{array}\right)\left(\begin{array}{cccc}
1 & 0 & 0 & 0 \\
0 & 0 & 1 & 0 \\
0 & 1 & y & 0 \\
0 & 0 & 0 & 1
\end{array}\right)\left(\begin{array}{cc}
a h f_{1} & 0 \\
0 & a h f_{2}
\end{array}\right)\right) \\
& \quad \times e^{-2 \rho}(a) e\left(-x_{1}-x_{3}\right) \delta_{\infty}\left(a h f_{3}\right) \psi(h) d x_{1} d x_{3} d y d h d u_{1} d u_{2} d a .
\end{aligned}
$$

The congruence

$$
\left(\begin{array}{cccc}
1 & x_{1} & u_{1} & 0 \\
0 & 1 & 0 & u_{2} \\
0 & 0 & 1 & x_{3} \\
0 & 0 & 0 & 1
\end{array}\right) \equiv\left(\begin{array}{cccc}
1 & 0 & u_{1} & 0 \\
0 & 1 & 0 & u_{2} \\
0 & 0 & 1 & 0 \\
0 & 0 & 0 & 1
\end{array}\right)\left(\begin{array}{cccc}
1 & x_{1} & 0 & 0 \\
0 & 1 & 0 & 0 \\
0 & 0 & 1 & x_{3} \\
0 & 0 & 0 & 1
\end{array}\right)
$$

modulo the center of $N$ implies that we can view the integral with respect to $d u_{1} d u_{2}$ as projecting $\tau^{0}$ to the trivial Fourier components with respect to those two variables, whereas the other integrations operate from the right. Right translation commutes with projection onto the trivial Fourier components, thus allowing us to shift the integration with respect to $d u_{1} d u_{2}$ all the way to the inside. The passage from $\tau$ to $\tau_{0}$ already involves a projection. Together with the $d u_{1} d u_{2}$-integral, this gives us the projection

$$
\tau \mapsto \tau_{\text {abelian }}, \quad \tau_{\text {abelian }}(g)=\int_{(\Gamma \cap[N, N]) \backslash N} \tau(n g) d n,
$$

onto the sum of the abelian Fourier coefficients - equivalently of invariants for the derived group $[N, N] \subset N$. Thus (5.37) reduces to

$$
\begin{aligned}
& P_{\nu}^{\Gamma_{0}}\left(A \tau, E_{\nu}\right)=2 \zeta(\nu+1) \times \\
& \quad \times \int_{A_{0}} \int_{G_{0}^{0}} \int_{\mathbb{R}} \int_{\mathbb{R}^{2} / \mathbb{Z}^{2}} \tau_{\text {abelian }}\left(\left(\begin{array}{cccc}
1 & x_{1} & 0 & 0 \\
0 & 1 & 0 & 0 \\
0 & 0 & 1 & x_{3} \\
0 & 0 & 0 & 1
\end{array}\right)\left(\begin{array}{cccc}
1 & 0 & 0 & 0 \\
0 & 0 & 1 & 0 \\
0 & 1 & y & 0 \\
0 & 0 & 0 & 1
\end{array}\right)\left(\begin{array}{cc}
a h f_{1} & 0 \\
0 & a h f_{2}
\end{array}\right)\right) \\
& \quad \times e^{-2 \rho}(a) e\left(-x_{1}-x_{3}\right) \delta_{\infty}\left(a h f_{3}\right) \psi(h) d x_{1} d x_{3} d y d h d a .
\end{aligned}
$$


Now we argue as we did in the passage from 4.27) to (4.35). First we substitute $e, n_{1}, s$ for $f_{1}, f_{2}, f_{3}$ as in (3.14). We then parameterize $h \in G_{0}^{0}$ as $h=k \tilde{a} n_{\tilde{x}}$, and observe that the argument of $\delta_{\infty}$ must lie in $N_{0} A_{0} s\{ \pm 1\}$ to give a non-zero contribution. At this point the argument diverges slightly from our earlier argument, where we worked modulo the center of $S L(2, \mathbb{R})$. There are three instances of the variable $h$ in $(5.39)$. When $h$ is replaced by $(-1) \cdot h$, $\delta_{\infty}$ remains unchanged, and the other two instances of $h$ effect a hypothetical sign change of $(-1)^{\eta_{1}+\eta_{2}+\eta_{3}+\eta_{4}}$ - hypothetical only since $\sum_{1<j<4} \eta_{j}=0$; cf. (5.12). Thus $k=e$ and $k=-1$ contribute equally, in effect doubling the factor 2 in (5.39). Since

$$
\left(\begin{array}{cccc}
1 & x_{1} & 0 & 0 \\
0 & 1 & 0 & 0 \\
0 & 0 & 1 & x_{3} \\
0 & 0 & 0 & 1
\end{array}\right)\left(\begin{array}{llll}
1 & 0 & 0 & 0 \\
0 & 0 & 1 & 0 \\
0 & 1 & y & 0 \\
0 & 0 & 0 & 1
\end{array}\right)\left(\begin{array}{cccc}
1 & \tilde{x} & 0 & 0 \\
0 & 1 & 0 & 0 \\
0 & 0 & 1 & \tilde{x} \\
0 & 0 & 0 & 1
\end{array}\right) \equiv\left(\begin{array}{cccc}
1 & -\tilde{x} y & 0 & 0 \\
0 & 1 & 0 & 0 \\
0 & 0 & 1 & \tilde{x} y \\
0 & 0 & 0 & 1
\end{array}\right)\left(\begin{array}{cccc}
1 & x_{1} & 0 & 0 \\
0 & 1 & 0 & 0 \\
0 & 0 & 1 & x_{3} \\
0 & 0 & 0 & 1
\end{array}\right)\left(\begin{array}{cccc}
1 & 0 & 0 & 0 \\
0 & 0 & 1 & 0 \\
0 & 1 & y & 0 \\
0 & 0 & 0 & 1
\end{array}\right)
$$

modulo a left factor lying in $[N, N]$, the variable $n_{\tilde{x}}$ can simply be absorbed into the $d x_{1} d x_{3}$-integration. We can therefore replace $\psi(h) d h$ by $e^{-2 \rho}(\tilde{a}) \psi_{A}(\tilde{a}) d \tilde{a}$ and the other instances of $h$ by $\tilde{a}$, as in 4.29 . The smoothing by $\psi$ has now been replaced by smoothing with respect to $\psi_{A}$, in the single variable $a$. This reflects the fact that the $A$-direction is the only noncompact direction for the integral 5.30), aside from the smoothing integral over $h \in G_{0}^{0}$, of cours $4^{5}$. Just as in section 4 4 , the smoothing in the variable $a$ will turn out to be unnecessary when we interpret the integrand as a distribution which can be made convergent by integration by parts - under our standing assumption that $\operatorname{Re} \nu \gg 0$. To summarize, we can eliminate the integration over $h$ and the factor $\psi(h)$ in (5.39), provided we double the factor 2 , set $h=e$ in the argument of $\tau^{0}$, and replace $\delta_{\infty}\left(a h f_{3}\right)$ by $\chi_{\nu+\rho}(a)$, in analogy to 4.28 and the comment that follows it. Finally we combine the factors $e^{-2 \rho}(a)$ and $\chi_{\nu+\rho}(a)$ into the single expression $\chi_{\nu-\rho}(a)$ :

$$
\begin{aligned}
& P_{\nu}^{\Gamma_{0}}\left(A \tau, E_{\nu}\right)=4 \zeta(\nu+1) \times \\
& \quad \times \int_{A_{0}} \int_{\mathbb{R}} \int_{\mathbb{R}^{2} / \mathbb{Z}^{2}} \tau_{\text {abelian }}\left(\left(\begin{array}{cccc}
1 & x_{1} & 0 & 0 \\
0 & 1 & 0 & 0 \\
0 & 0 & 1 & x_{3} \\
0 & 0 & 0 & 1
\end{array}\right)\left(\begin{array}{cccc}
1 & 0 & 0 & 0 \\
0 & 0 & 1 & 0 \\
0 & 1 & y & 0 \\
0 & 0 & 0 & 1
\end{array}\right)\left(\begin{array}{cc}
a & 0 \\
0 & a n_{1}
\end{array}\right)\right) \\
& \quad \times \chi_{\nu-\rho}(a) e\left(-x_{1}-x_{3}\right) d x_{1} d x_{3} d y d a .
\end{aligned}
$$

For each $n \in(\mathbb{Z}-\{0\})^{3}$, there exists a unique $B_{n, \mu, \eta} \in W_{\mu, \eta}^{-\infty}$ characterized by the properties

$$
\begin{gathered}
\pi_{\mu, \eta}(n(x, u, v)) B_{n, \mu, \eta}=e\left(-n_{1} x_{1}-n_{2} x_{2}-n_{3} x_{3}\right) B_{n, \mu, \eta} \\
B_{n, \mu, \eta}(n(x, u, v))=e\left(n_{1} x_{1}+n_{2} x_{2}+n_{3} x_{3}\right)
\end{gathered}
$$

5]; these identities are analogous to 4.19 and use the notation (5.16). The $B_{n, \mu, \eta}$ corresponding to different values of $n$ are related by the action of the

\footnotetext{
${ }^{5}$ The integration with respect to $y \in \mathbb{R}$ in the equivalent integral 5.39 was ob-
} tained by unfolding an integral over $\mathbb{R} / \mathbb{Z}$. 
diagonal subgroup $A \subset G$, but this need not concern us here. The cuspidality of $\tau$ implies that the Fourier coefficients in (5.17) vanish whenever one or more of the indices are zero. Explicitly,

$$
a_{n} \neq 0 \Longrightarrow n \in(\mathbb{Z}-\{0\})^{3} .
$$

Comparing (5.42) to (5.16) and the definition 5.38 of $\tau_{\text {abelian }}$, one finds

$$
\tau_{\text {abelian }}=\sum_{n \in(\mathbb{Z}-\{0\})^{3}} a_{n} B_{n, \mu, \eta} .
$$

The inner integral in 5.41 picks out the terms in the sum corresponding to $n_{1}=n_{3}=1$. Hence

$$
\begin{aligned}
& P_{\nu}^{\Gamma_{0}}\left(A \tau, E_{\nu}\right)=4 \zeta(\nu+1) \times \\
& \times \sum_{\ell \neq 0} a_{1, \ell, 1} \int_{A_{0}} \int_{\mathbb{R}} B_{1, \ell, 1 ; \mu, \eta}\left(\left(\begin{array}{cccc}
1 & 0 & 0 & 0 \\
0 & 0 & 1 & 0 \\
0 & 1 & 0 \\
0 & 0 & 0 & 1
\end{array}\right)\left(\begin{array}{cc}
a & 0 \\
0 & a n_{1}
\end{array}\right)\right) \chi_{\nu-\rho}(a) d y d a .
\end{aligned}
$$

We parameterize $A_{0}$ as in $4.19 p, A_{0}=\left\{a_{t} \mid t \in \mathbb{R}\right\}$. Then $\chi_{\nu-\rho}\left(a_{t}\right)=$ $e^{(\nu-1) t}$; cf. 4.30). Conjugating $a_{t}$ across $n_{1}$ and using the transformation rule (5.9) that defines $W_{\mu, \eta}^{-\infty}$, we can re-write 5.45) as follows:

$$
\begin{aligned}
& P_{\nu}^{\Gamma_{0}}\left(A \tau, E_{\nu}\right)=4 \zeta(\nu+1) \sum_{\ell \neq 0} a_{1, \ell, 1} \times \\
& \quad \times \int_{\mathbb{R}^{2}} B_{1, \ell, 1 ; \mu, \eta}\left(\left(\begin{array}{cccc}
1 & 0 & 0 \\
0 & 0 & 0 & 0 \\
0 & 1 & 0 & 0 \\
0 & 0 & 0 & 1
\end{array}\right)\left(\begin{array}{cc}
1 & 0 \\
0 & a_{t} n_{1} a_{-t}
\end{array}\right)\right) e^{\left(\nu+1-2 \mu_{1}-2 \mu_{3}\right) t} d y d t .
\end{aligned}
$$

The passage from (5.45) to (5.46) also depends on the identity (5.13), which implies $\left(1-\mu_{1}+\mu_{2}\right)+\left(1-\mu_{3}+\mu_{4}\right)=2-2\left(\mu_{1}+\mu_{3}\right)$. Note that $a_{t} n_{1} a_{-t}=n_{e^{2 t}}$; cf. (3.17). We appeal to the matrix identity

$$
\left(\begin{array}{llll}
1 & 0 & 0 & 0 \\
0 & 0 & 1 & 0 \\
0 & 1 & y & 0 \\
0 & 0 & 0 & 1
\end{array}\right)\left(\begin{array}{llll}
1 & 0 & 0 & 0 \\
0 & 1 & 0 & 0 \\
0 & 0 & 1 & z \\
0 & 0 & 0 & 1
\end{array}\right)=\left(\begin{array}{cccc}
1 & 0 & 0 & 0 \\
0 & 1 & 1 / y & z \\
0 & 0 & 1 & y z \\
0 & 0 & 0 & 1
\end{array}\right)\left(\begin{array}{cccc}
1 & 0 & 0 & 0 \\
0 & -1 / y & 0 & 0 \\
0 & 1 & y & 0 \\
0 & 0 & 0 & 1
\end{array}\right),
$$

with $z=e^{2 t}$, the characterization 5.42 of $B_{n, \mu, \eta}$, and once more to 5.9 , to conclude

$$
\begin{aligned}
& P_{\nu}^{\Gamma_{0}}\left(A \tau, E_{\nu}\right)=4(-1)^{\eta_{2}} \zeta(\nu+1) \sum_{\ell \neq 0} a_{1, \ell, 1} \times \\
& \quad \times \int_{\mathbb{R}^{2}} e\left(\ell / y+y e^{2 t}\right)(\operatorname{sgn} y)^{\eta_{2}+\eta_{3}}|y|^{\mu_{2}-\mu_{3}-1} e^{\left(\nu+1-2 \mu_{1}-2 \mu_{3}\right) t} d y d t .
\end{aligned}
$$

We simplify the integrand by making the change of variables $y \mapsto \ell / y$, followed by the substitution $x=|\ell||y|^{-1} e^{2 t}$. Then $d x=2 x d t$, hence

$$
\begin{aligned}
& P_{\nu}^{\Gamma_{0}}\left(A \tau, E_{\nu}\right)=2(-1)^{\eta_{2}} \zeta(\nu+1) \sum_{\ell \neq 0} a_{1, \ell, 1}|\ell|^{\mu_{1}+\mu_{2}-\frac{\nu+1}{2}} \times \\
& \quad \times \int_{0}^{\infty} \int_{\mathbb{R}} \frac{e(y+(\operatorname{sgn} \ell y) x)}{(\operatorname{sgn} \ell y)^{\eta_{2}+\eta_{3}}} x^{\frac{\nu-1}{2}-\mu_{1}-\mu_{3}}|y|^{\frac{\nu-1}{2}-\mu_{1}-\mu_{2}} d y d x .
\end{aligned}
$$


Recall the definition 5.20 of the exterior square $L$-function. We now separate the terms in $(5.49)$ corresponding to positive and negative values of $\ell$. Appealing to (5.12) and (5.18), we find

$$
\begin{aligned}
& P_{\nu}^{\Gamma_{0}}\left(A \tau, E_{\nu}\right)=2 L\left(\frac{\nu+1}{2}, \tau, E x t^{2}\right) \times \\
& \quad \times\left\{(-1)^{\eta_{2}} \int_{0}^{\infty} \int_{\mathbb{R}} \frac{e(y+(\operatorname{sgn} y) x)}{(\operatorname{sgn} y)^{\eta_{2}+\eta_{3}}}|x|^{\frac{\nu-1}{2}-\mu_{1}-\mu_{3}}|y|^{\frac{\nu-1}{2}-\mu_{1}-\mu_{2}} d y d x\right. \\
& \left.\quad+(-1)^{\eta_{4}} \int_{-\infty}^{0} \int_{\mathbb{R}} \frac{e(y+(\operatorname{sgn} y) x)}{(\operatorname{sgn} y)^{\eta_{2}+\eta_{3}}}|x|^{\frac{\nu-1}{2}-\mu_{1}-\mu_{3}}|y|^{\frac{\nu-1}{2}-\mu_{1}-\mu_{2}} d y d x\right\} .
\end{aligned}
$$

The factor in curly parentheses equals

$$
\begin{aligned}
& \int_{\mathbb{R}^{2}} \frac{(-1)^{\eta_{2}} e(x+y)}{(\operatorname{sgn} x)^{\eta_{1}+\eta_{3}}(\operatorname{sgn} y)^{\eta_{1}+\eta_{2}}}|x|^{\frac{\nu-1}{2}-\mu_{1}-\mu_{3}}|y|^{\frac{\nu-1}{2}-\mu_{1}-\mu_{2}} d y d x= \\
& \quad=\int_{\mathbb{R}} \frac{(-1)^{\eta_{2}} e(x)}{(\operatorname{sgn} x)^{\eta_{1}+\eta_{3}}}|x|^{\frac{\nu-1}{2}-\mu_{1}-\mu_{3}} d x \times \int_{\mathbb{R}} \frac{e(y)}{(\operatorname{sgn} y)^{\eta_{1}+\eta_{2}}}|y|^{\frac{\nu-1}{2}-\mu_{1}-\mu_{2}} d y \\
& =(-1)^{\eta_{2}} G_{\eta_{1}+\eta_{3}}\left(\frac{\nu+1}{2}-\mu_{1}-\mu_{3}\right) G_{\eta_{1}+\eta_{2}}\left(\frac{\nu+1}{2}-\mu_{1}-\mu_{2}\right) .
\end{aligned}
$$

That completes the proof of the lemma.

\section{References}

[1] Daniel Bump, The Rankin-Selberg method: a survey, Number theory, trace formulas and discrete groups (Oslo, 1987), 1989, pp. 49-109.

[2] Daniel Bump and Solomon Friedberg, The exterior square automorphic $L$ functions on $\mathrm{GL}(n)$, Festschrift in honor of I. I. Piatetski-Shapiro on the occasion of his sixtieth birthday, Part II (Ramat Aviv, 1989), 1990, pp. 47-65.

[3] W. Casselman, Jacquet modules for real reductive groups, Proceedings of the International Congress of Mathematicians (Helsinki, 1978), 1980, pp. 557-563.

[4] Canonical extensions of Harish-Chandra modules to representations of $G$, Canad. J. Math. 41 (1989), no. 3, 385-438.

[5] William Casselman, Henryk Hecht, and Dragan Miličić, Bruhat filtrations and Whittaker vectors for real groups, The mathematical legacy of Harish-Chandra (Baltimore, MD, 1998), 2000, pp. 151-190.

[6] Hervé Jacquet, Automorphic forms on GL(2). Part II, Springer-Verlag, Berlin, 1972. Lecture Notes in Mathematics, Vol. 278.

[7] Hervé Jacquet and Joseph Shalika, Exterior square L-functions, Automorphic forms, Shimura varieties, and $L$-functions, Vol. II (Ann Arbor, MI, 1988), 1990, pp. 143-226.

[8] Henry H. Kim, Langlands-Shahidi method and poles of automorphic Lfunctions: application to exterior square L-functions, Canad. J. Math. 51 (1999), no. 4, 835-849.

[9] Functoriality for the exterior square of $\mathrm{GL}_{4}$ and the symmetric fourth of $\mathrm{GL}_{2}$, J. Amer. Math. Soc. 16 (2003), no. 1, 139-183. With appendix 1 by Dinakar Ramakrishnan and appendix 2 by Kim and Peter Sarnak. 
[10] R. P. Langlands, Problems in the theory of automorphic forms, Lectures in modern analysis and applications, III, 1970, pp. 18-61. Lecture Notes in Math., Vol. 170.

[11] Robert P. Langlands, Euler products, Yale University Press, New Haven, Conn., 1971.

[12] Hans Maass, Über eine neue Art von nichtautomorphen analytische Funktionen und die Bestimmung Dirichletscher Reihen durch Funktionalgleichungen, Math. Annalen 121 (1949), 141-183.

[13] Stephen D. Miller and Wilfried Schmid, Distributions and analytic continuation of Dirichlet series, J. Funct. Anal. 214 (2004), no. 1, 155-220.

[14] _ Automorphic Distributions, L-functions, and Voronoi Summation for $G L(3)$, Annals of Mathematics. To appear.

$[15] \_$, The archimedean theory of the Exterior Square L-functions over $\mathbb{Q}$. preprint, 2005.

[16] A. I. Oksak, Trilinear Lorentz invariant forms, Comm. Math. Phys. 29 (1973), 189-217.

[17] R. A. Rankin, Contributions to the theory of Ramanujan's function $\tau(n)$ and similar arithmetical functions. I. The zeros of the function $\sum_{n=1}^{\infty} \tau(n) / n^{s}$ on the line $\mathfrak{R} s=13 / 2$. II. The order of the Fourier coefficients of integral modular forms, Proc. Cambridge Philos. Soc. 35 (1939), 351-372.

[18] Atle Selberg, Bemerkungen über eine Dirichletsche Reihe, die mit der Theorie der Modulformen nahe verbunden ist, Arch. Math. Naturvid. 43 (1940), 47-50 (German).

[19] Freydoon Shahidi, A proof of Langlands' conjecture on Plancherel measures; complementary series for p-adic groups, Ann. of Math. (2) 132 (1990), no. 2, $273-330$

[20] Takuro Shintani, On an explicit formula for class-1 "Whittaker functions" on $G L_{n}$ over P-adic fields, Proc. Japan Acad. 52 (1976), no. 4, 180-182.

[21] Eric Stade, Mellin transforms of $\mathrm{GL}(n, \mathbb{R})$ Whittaker functions, Amer. J. Math. 123 (2001), no. 1, 121-161.

[22] Nolan R. Wallach, Asymptotic expansions of generalized matrix entries of representations of real reductive groups, Lie group representations, I (College Park, Md., 1982/1983), 1983, pp. 287-369. 